\title{
The nature of self-regulation in photosynthetic light-harvesting antenna
}

\author{
Jevgenij Chmeliov ${ }^{1,2, \dagger}$, Andrius Gelzinis ${ }^{1,2, \dagger}$, Egidijus Songaila $^{2}$, Ramūnas Augulis $^{2}$, Christopher D. P. Duffy ${ }^{3}$, \\ Alexander V. Ruban ${ }^{3}$, and Leonas Valkunas ${ }^{1,2, *}$ \\ ${ }^{1}$ Department of Theoretical Physics, Faculty of Physics, Vilnius University, Saulètekio Ave. 9, LT-10222 Vilnius, Lithuania \\ ${ }^{2}$ Department of Molecular Compound Physics, Center for Physical Sciences and Technology, Savanoriụ Ave. 231, LT-02300 Vilnius, Lithuania \\ ${ }^{3}$ The School of Biological and Chemical Sciences, Queen Mary, University of London, Mile End Road, London E1 4NS, U.K.
}

Photosynthesis is the key natural process performed by green plants, algae, and photosynthetic bacteria, during which solar radiation is used to supply energy to Earth's biosphere. ${ }^{1}$ The lightharvesting systems of photosynthetic organisms exhibit an un5 precedented quantum efficiency: up to $99 \%$ of the absorbed photons are successfully utilised during charge separation in the reaction centres ( $R C s$ ) of the photosynthetic units. ${ }^{2}$ Meanwhile, intense sunlight may result in over-excitation of the light-harvesting antenna and the formation of reactive compounds capable of "burn10 ing out" the whole photosynthetic apparatus. To avoid any possible photodamage, plants have developed several self-regulatory mechanisms. The most efficient one, operating on a molecular level and dissipating the detrimental excess excitation as heat, reversibly activates and relaxes within several seconds to minutes and is com15 monly known as a non-photochemical quenching (NPQ). ${ }^{3}$ While it is believed to take place in the plant's major light harvesting complexes (LHCII), there is still no consensus regarding its physical origin. ${ }^{4-8}$ Here we have revealed the self-regulatory nature of light harvesting control by identifying several functional states of 20 the LHCII complexes, one of which is responsible for quenching, and connect them with the underlying molecular mechanisms. We present high-resolution time-resolved fluorescence measurements of LHCII trimers and their aggregates, performed over a wide temperature range. Based on simulations of the excitation en25 ergy transfer in the LHCII aggregate, we associate the red-emitting state, having fluorescence maximum at $\sim 700 \mathrm{~nm}$, with the partial mixing of excitonic and chlorophyll-chlorophyll charge transfer states. On the other hand, the quenched state has a totally different nature and is related to the incoherent excitation transfer to 30 the short-lived carotenoid excited states. Our results show that the required level of photoprotection in vivo can be achieved by a very subtle change in the number of LHCIIs switched to the quenched state.

The process of NPQ provides a short-term adaptation by controlling 35 the excitation energy flow within the photosynthetic thylakoid membranes. Its activation depends on variations of the transmembrane $\Delta \mathrm{pH}$ that correlates with the rate of photochemistry in RCs and therefore with the incoming solar radiation. NPQ manifests itself by a strongly reduced chlorophyll (Chl) fluorescence lifetime and is accompanied by

40 the clustering and/or increased energetic inter-connectivity of the lightharvesting antenna. ${ }^{9,10}$ The currently proposed models attribute NPQ to a formation of either Chl-Chl ${ }^{11}$ or Chl-carotenoid ${ }^{4}$ (Car) charge transfer (CT) state, a coherent mixing between Chls and short-lived dark $S_{1}$ state of Cars, ${ }^{12,13}$ or simple incoherent excitation energy transfer from Chls to nearby Cars. ${ }^{5,6}$

Although the studies of intact thylakoid membranes provide direct

\footnotetext{
*Corresponding author: leonas.valkunas@ ff.vu.lt.

${ }^{\dagger}$ These authors contributed equally to this work.
}

possibility to observe NPQ, identification of the physical NPQ mechanism becomes hardly possible due to the presence of RCs and both types of photosystems (PS), PSI and PSII, that affect the spectroscopic signatures and the kinetics of the quenched samples. On the other hand, in vitro studies of the purified LHCII complexes have revealed many characteristic properties of the light-harvesting processes happening in vivo. First of all, aggregation of LHCIIs, observed at low detergent concentrations, results in a considerable excitation energy dissipation. ${ }^{14,15}$ Measurements, performed on LHCII aggregates ${ }^{16,17}$ and LHCII crystals ${ }^{5}$ at the temperatures below $100 \mathrm{~K}$, have additionally demonstrated the appearance of a new strong peak in the spectral region around $700 \mathrm{~nm}$ of the steady-state fluorescence spectrum, resembling red-shifted chlorophyll fluorescence in the $77 \mathrm{~K}$ thylakoid membranes. ${ }^{14,15,18}$ Also, freeze fracture electron microscopy revealed clustering/aggregation patters of the LHCII complexes on the protoplasmic fracture face of the stacked membranes in NPQ state. ${ }^{18}$ All this suggests that NPQ is related to the aggregation of the light-harvesting complexes, even though similarities between the in vitro aggregates and thylakoid membranes does not guarantee that their behaviour is exactly the same. Therefore, by studying the two limiting cases of the "pristine" light-harvesting antenna-single LHCII trimers and their large aggregates - the obtained results can be generalised to the in vivo systems, which, besides the PSII core and the minor antenna complexes, are composed of small clusters of about 3-4 LHCII trimers per RC. ${ }^{2}$

To examine excitation energy dynamics in LHCII aggregate and its relationship to NPQ, in the current work we performed time-resolved fluorescence measurements of high temporal resolution by taking advantage of the streak-camera-based spectrograph that enabled simultaneous temporal and spectral decomposition of the fluorescence signal. Comparing with other non-linear spectroscopic techniques, fluorescence measurements, performed under low excitation conditions. are simpler to interpret as they are not affected by the non-linear annihilation processes. Since the proposed mechanisms of NPQ are expected to demonstrate different behaviour at various temperatures, we measured fluorescence signal over a wide temperature range from $273 \mathrm{~K}$ down to $15 \mathrm{~K}$. A typical time-resolved fluorescence spectrum, observed at $150 \mathrm{~K}$ temperature, is shown in Figure 1a as a two-dimensional map and reveals a notable difference of the fluorescence decay kinetics at various wavelengths (compare, e.g., vertical slices at 680, 700, and $720 \mathrm{~nm}$, shown in Figure 1b). Fluorescence kinetics at longer wavelengths exhibit much slower decay (but still faster than in nonaggregated LHCIIs, $c f$. Extended Data Figure 1), so in spite of their small initial amplitude, their corresponding spectral region starts to dominate after several nanoseconds following the initial excitation, see Figure 1c. As a result, in the course of time in addition to the $680 \mathrm{~nm}$ peak, another peak at $\sim 710 \mathrm{~nm}$ appears, resulting in a typical wing in the far red region of the steady-state fluorescence spectra of LHCII aggregates (Figure 1d), though LHCII absorption spectra show no notable 

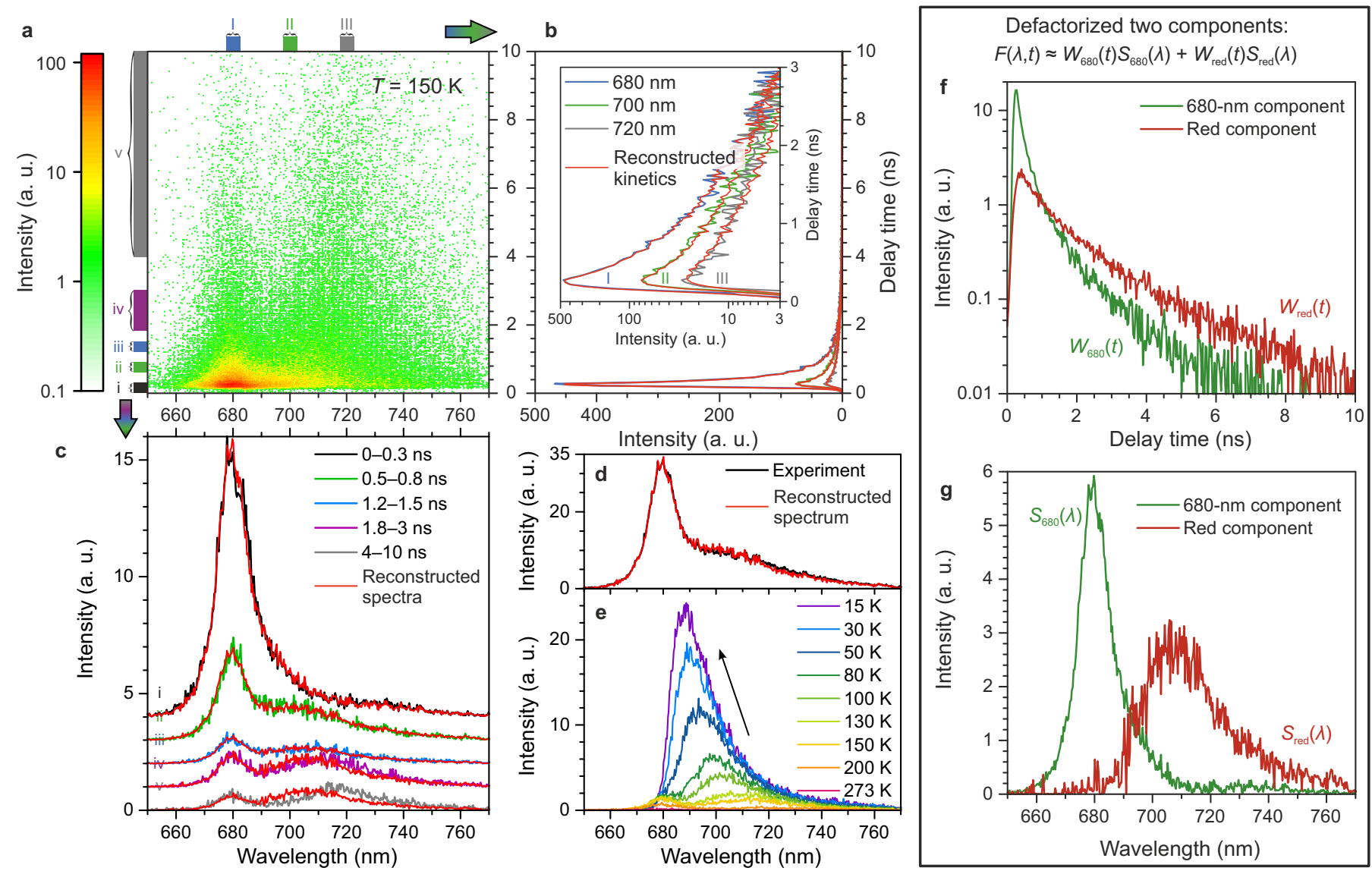

Figure 1 I Time-resolved fluorescence measurements of LHCII aggregates at $150 \mathrm{~K}$ temperature. a, Two-dimensional plot illustrating evolution of fluorescence intensity $F(\lambda, t)$. $\mathbf{b}$, Vertical slices (fluorescence kinetics) of panel (a) obtained by integrating fluorescence intensity map over a 5 -nm-wide regions around 680,700, and $720 \mathrm{~nm}$ (indicated with colour bars I-III). Inset, the same kinetics on a semi-logarithmic scale. c, Horizontal slices (fluorescence spectra) of panel (a) obtained by integrating fluorescence intensity map over different time intervals (indicated with colour bars $\mathrm{i}-\mathrm{v}$ ). The initially strong fluorescence signal at $680 \mathrm{~nm}$ exhibits a fast decay, providing an opportunity to detect slowly decaying signal at 700-nm spectral region. d, Steady-state fluorescence spectrum of LHCII aggregate, obtained by integrating fluorescence intensity map from panel (a) over the whole time region. e, Integrated fluorescence spectra over 1.8-3 ns delay times, measured at various temperatures and revealing the blue shift of the red-emitting band with the temperature drop (indicated with arrow). Panels $\mathbf{f}$ and $\mathbf{g}$ depict the fluorescence decay kinetics and steady-state fluorescence spectra, respectively, of two major components that were extracted from intensity map in panel (a) by means of multivariate curve resolution (see equation above panel (f)) and correspond to two distinct emitting states in LHCII aggregates. Red lines in panels (b), (c), and (d) illustrate corresponding fluorescence kinetics and spectra, reconstructed from the sum of these two components, see text and Methods for details.

differences upon aggregation ( $c f$. Extended Data Figure 2). Similar results were obtained at other temperatures as well, see Extended Data Figures 3 and 4 . These results indicate an effective and only slightly reversible excitation transfer from the whole aggregate into the centres responsible for the long-lived red-shifted Chl fluorescence. Since the maximum amplitude of the excitation kinetics at $700 \mathrm{~nm}$ is almost an order of magnitude smaller than that of the dominating spectral component at $680 \mathrm{~nm}$ while its decay rate is considerably slower (meaning back-transfer from the red states to the 680 -nm ones is rather slow), not all the excitations reach these specific "red" centres. Thus additional excitation "quenching" centres should co-exist within the aggregate. These observations are in line with single-molecule spectroscopy (SMS) experiments, ${ }^{19}$ where it was shown that on a micro- to millisecond timescale the same LHCII trimer can reversibly switch between several spectrally different states: although most of the time the fluorescence spectrum exhibited a strong peak at $680 \mathrm{~nm}$, sometimes the position of this peak shifted to $\sim 700 \mathrm{~nm}$, or the fluorescence signal was disappearing at all.

We analysed the experimental results in terms of a multivariate curve resolution $^{20}$ (see equation above Figure 1f and Methods for details), which allowed us to describe our data with a minimum number of fluorescing states, each having a clear physical interpretation. To fit the whole time-resolved fluorescence map, we used a time-dependent weighted sum of only two spectral components, one describing the dominating LHCII species fluorescing at $680 \mathrm{~nm}$, and another corresponding to the LHCIIs being in the red state. The obtained bestfitted fluorescence spectra of these components $\left(S_{680}(\lambda)\right.$ and $\left.S_{\text {red }}(\lambda)\right)$ for $150 \mathrm{~K}$ are presented in Figure $1 \mathrm{~g}$, and their kinetics $\left(W_{680}(t)\right.$ and $\left.W_{\text {red }}(t)\right)$ are shown in Figure 1f. The quality of such spectral decomposition was validated by reconstructing total fluorescence kinetics $F(\lambda, t)$ at various wavelengths and fluorescence spectra at different time delays, see red lines in Figures $1 b-d$. Fluorescence from LHCII aggregates at other temperatures was analysed in a similar way (see Methods and Extended Data Figures 3-4 for details), and the obtained results are summarised in Figure 2. The fluorescence lineshapes of both the $680-\mathrm{nm}$ and the red components become notably narrower at lower temperatures, as expected, though in all cases the spectrum of the red component is much broader. More important is the fact that while the peak maximum of the $680 \mathrm{~nm}$ component stays roughly at the same position at all considered temperatures, its counterpart shows 

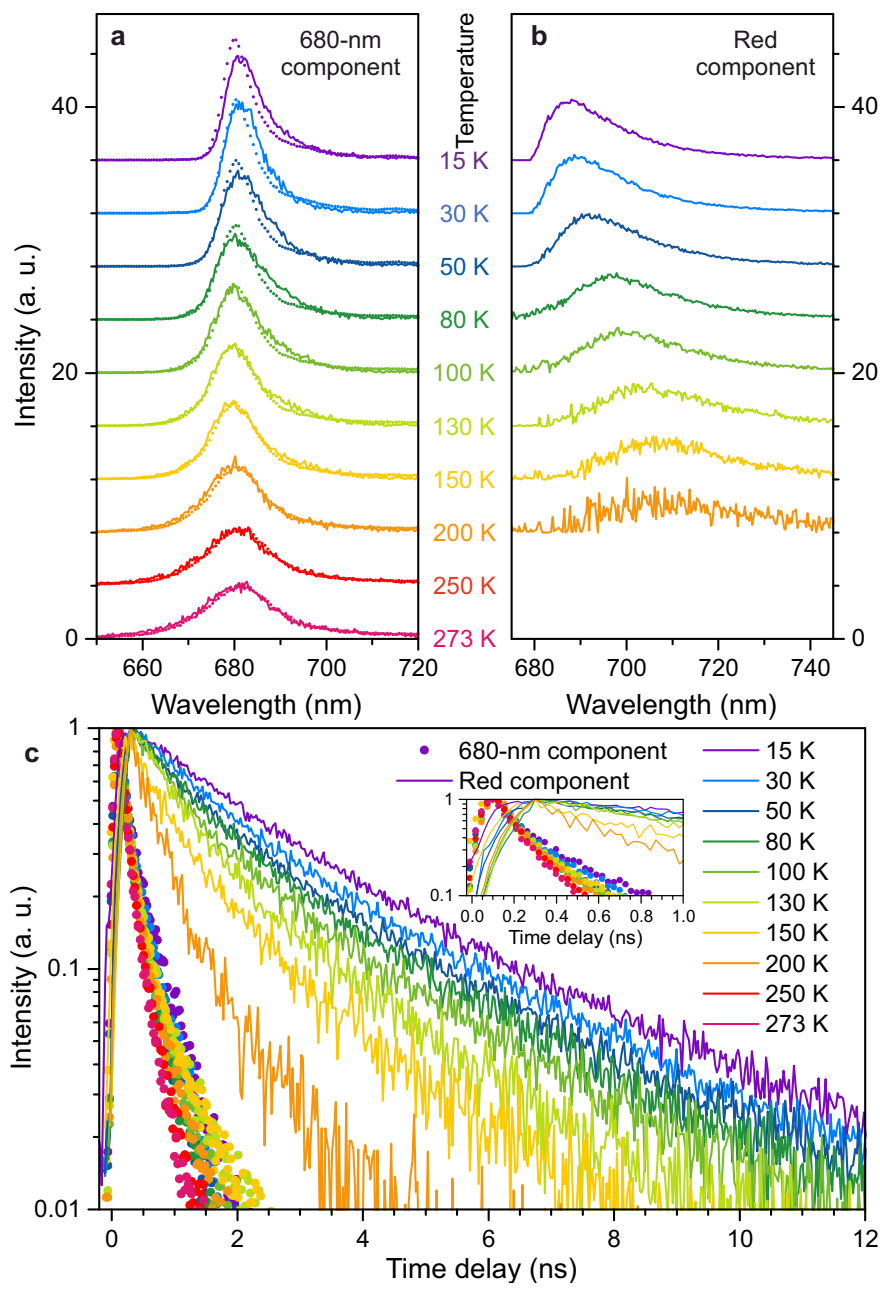

Figure 2 । Results of the decomposing time-resolved fluorescence spectra of LHCII aggregates, measured at various temperatures, into two major differently emitting components. a and $\mathbf{b}$, temperature-dependent fluorescence spectra of the extracted $680-\mathrm{nm}$ and red components, respectively. Spectra at different temperatures are shifted vertically for clarity. For comparison, dots in panel (a) represent the fluorescence spectra, obtained from the measurements of solubilised LHCII trimers (see also Extended Data Figure 1). At $250 \mathrm{~K}$ and $273 \mathrm{~K}$ temperatures, only one spectral component was sufficient to reproduce experimental results. c, normalised fluorescence decay kinetics of the decomposed two components at various temperatures. Inset, the enlarged initial part of the decay kinetics.

considerable blue shift upon cooling: from $\sim 707 \mathrm{~nm}$ at $200 \mathrm{~K}$ down to $688 \mathrm{~nm}$ at $15 \mathrm{~K}$ (note that this effect is also visible in the raw data, see Figure 1e). The shift results in a very strong overlap of the spectra of both components and the disappearance of the second peak in the steady-state fluorescence spectra of LHCII aggregates under temperatures below $50 \mathrm{~K} .{ }^{16,17}$ As a control, time-resolved fluorescence spectra of LHCII trimers prior their aggregation were also measured and analysed (see Methods and Extended Data Figure 1). Their obtained fluorescence spectra (shown with the dotted lines in Figure 2a) were found to follow those of the dominating 680-nm component in LHCII aggregates, further supporting the applied decomposition method. Thi also implies that relatively few complexes are in the red-emitting state.

The obtained fluorescence decay kinetics of both components show non-exponential behaviour (see Figure 2c), which should be due to inhomogeneity of the number of complexes in the red or quenched state (this corresponds to the fluctuating antenna model, ${ }^{21}$ see also below)
Interestingly, fluorescence kinetics of the red LHCII species exhibited much slower decay rate and much more pronounced temperature dependence in comparison with those of the 680-nm component. This effect clearly indicates very slow excitation back-transfer rate from the red to the dominating LHCII species that we proposed earlier from the simple analysis of the fluorescence intensity map-in the opposite case, the decay rate of the kinetics of both components would be comparable. Secondly, the fluorescence of the red component reaches its maximum about 200 ps later than the fluorescence of the 680-nm component (see inset in Figure 2c). This means that just after the initial excitation the total energy population of the red LHCIIs still increases due to incoming excitation energy transfer from the nearby complexes. Such behaviour, however, contradicts the so-called excitation radicalpair equilibrium model, which postulates infinitely fast excitation equilibration over the whole photosynthetic antenna ${ }^{22}$ or across an artificial LHCII aggregate, ${ }^{11}$ and provides an independent support for the diffusion-limited regime of excitation energy dynamics.

To extract more details from the data, we performed simulations of excitation energy transfer using the simplest model of LHCII aggregate, which is schematically shown in Figure $3 \mathrm{a}-\mathrm{b}$. Based on the trimeric nature of LHCII complexes, their most favourable arrangement in the aggregate should resemble a hexagonal lattice, that was indeed observed in earlier studies by means of electron microscopy. ${ }^{23}$ By combining the above qualitative analysis and the current knowledge from the SMS measurements of single LHCII complexes, ${ }^{19}$ we assume that at any given time each LHCII monomer can be in one of the 3 possible distinct states discussed above-the most favourable one (corresponding to the 680-nm component), the red-emitting one, or the quenched one. An even simpler two-state model, when the redemitting state is also responsible for the excitation quenching, did not lead to any adequate reproduction of the experimental data. Since it takes just several picoseconds for the excitation to equilibrate within a monomer, ${ }^{24-26}$ we neglected this process and analysed the whole aggregate in terms of the coarse-grained model ${ }^{27,28}$ by implicitly taking into account only excitation hopping between the adjacent monomers. With these hopping rates being treated as fitting parameters, the total excitation population dynamics of the 680-nm-emitting and red complexes was calculated and then averaged over random distribution of the red and quenched complexes within the aggregate as well as the actual numbers of these specific complexes per aggregate (see Methods for details). This accounts for fluctuating properties of the antenna. The obtained mean excitation decay kinetics were then fitted to the decomposed fluorescence kinetics of the 680-nm and red components, resulting in a good reproduction of all the experimental data (see Figure $3 \mathrm{c}$ for $15 \mathrm{~K}, 100 \mathrm{~K}$, and $273 \mathrm{~K}$ measurements and Extended Data Figure $6 \mathrm{a}$ for other temperatures). The obtained model parameters and their temperature dependence are given in Extended Data Figure 6b-d. The timescales of excitation transfer between neighbouring complexes being in different states as well as mean excitation relaxation times, obtained at various temperatures, are also summarised in Figure $3 \mathrm{~b}$. For further verification of the model, the same calculated kinetics of the 680-nm species were re-convolved with a much shorter instrument response function, and the obtained results closely matched the independently measured initial part of fluorescence decay, as shown in Extended Data Figure 7.

Our measurements and simulations revealed several important features of the red-emitting states: (i) their fluorescence spectra (Figure $2 \mathrm{~b}$ ) are much broader than those of the dominating 680-nmemitting states and exhibit a considerable blue shift upon temperature decrease, (ii) the mean concentration of the red complexes strongly varies with temperature ( $c f$. inset in Figure $3 \mathrm{c}$ ), and (iii) the fluorescence from the red states decays on a nanosecond timescale ( $c f$. Figure 2c). This allows us to make conclusions about the molecular 

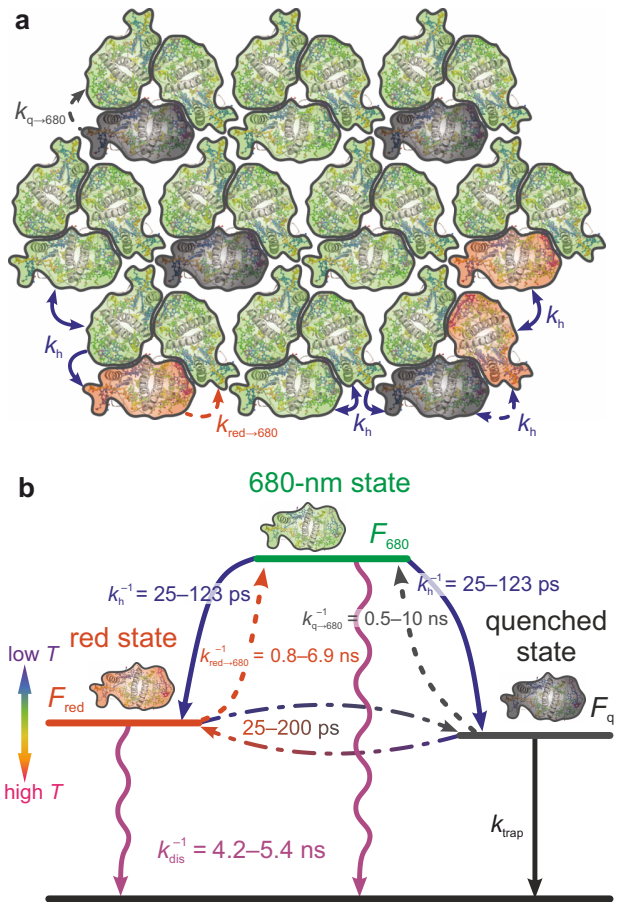

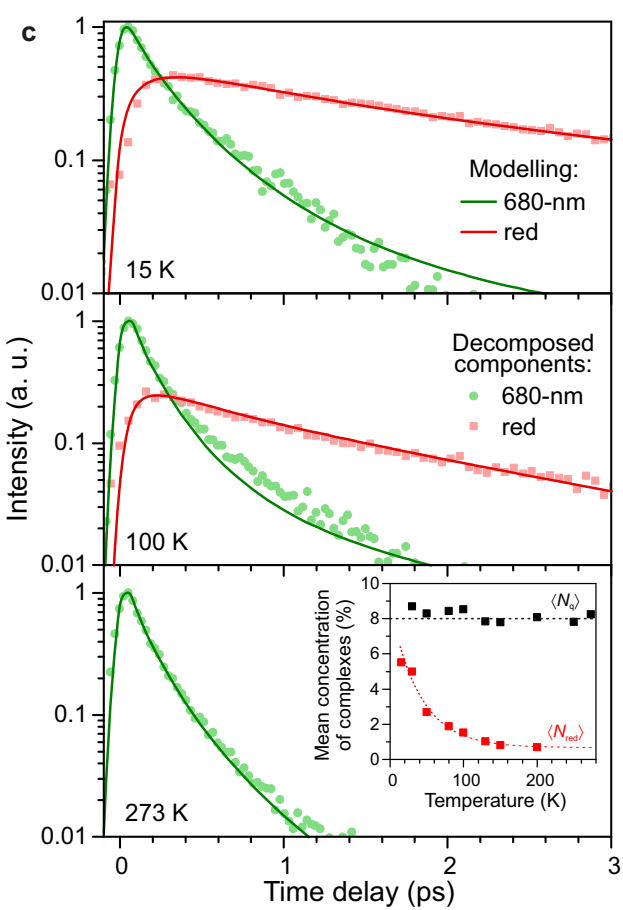

d
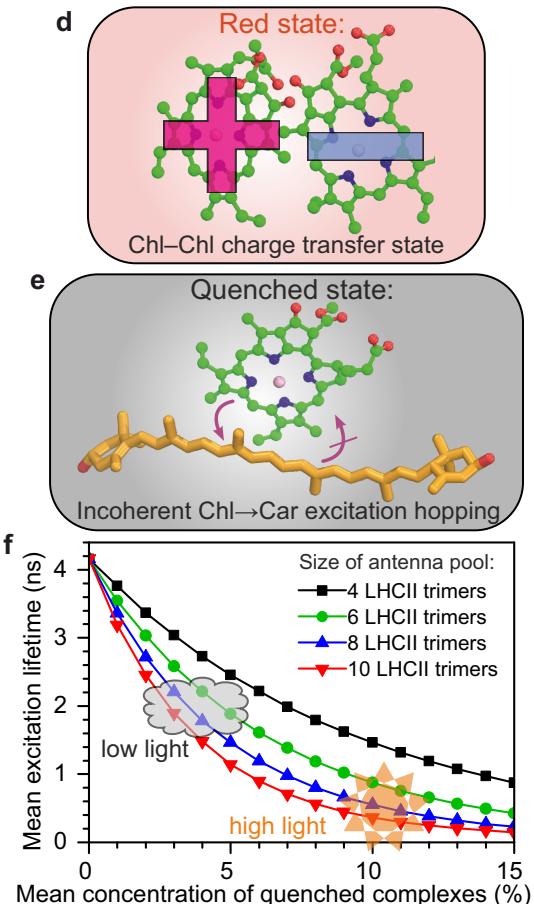

Figure 3 I Model for excitation energy transfer in LHCII aggregate. a, proposed hexagonal arrangement of the LHCII monomers in the aggregate. Depending on the conformational state of its protein, each LHCII monomer can be in one of the three different states-the 680-nmemitting one (the most probable, indicated with green), the red-emitting one (indicated with red), and the quenched one (indicated with grey). Inter-monomer excitation hopping rates are indicated with arrows. b, schematic energy level diagram of different states of LHCII monomers. Possible transitions and excitation transfer routes between neighbouring complexes are indicated with arrows, the corresponding numerical values present the timescales of these transitions at various temperatures, as obtained from the analysis of the experimental data and our simulations. Any value of $k_{\text {trap }}^{-1}$ from 25 to 100 ps resulted in very similar fluorescence kinetics, although intermediate values of $\sim 50$ ps produced the smallest mean square error of the fitted kinetics. At lower temperatures, the free energy of the red-emitting state $\left(F_{\text {red }}\right)$ was found to approach that of the 680-nm-emitting state $\left(F_{680}\right)$, as indicated with the multi-colour arrow on the left. See also Methods for details. c, best-fitted fluorescence kinetics at 3 particular temperatures. Inset, the obtained temperature dependence of the mean concentration of LHCII complexes per aggregate (in $\%$ ) being either in the red-emitting $\left(\left\langle N_{\text {red }}\right\rangle\right)$ or quenched $\left(\left\langle N_{\mathrm{q}}\right\rangle\right)$ state. $\mathbf{d}$ and $\mathbf{e}$, physical origin of the red-emitting and quenched states, respectively, as determined from our modelling results. f, calculated dependence of the mean excitation lifetime on the mean concentration of the quenched monomers in the small variously sized LHCII aggregates resembling the size of light-harvesting antenna of PSII in intact thylakoid membranes. Shaded regions correspond to the low-light (mean excitation lifetime when all RCs are closed is about $2 \mathrm{ns)}$ and high-light conditions (additional $\sim 3-4$-fold drop of the fluorescence quantum yield), respectively.

mechanism that is responsible for the whole complex being in a redemitting state. The first observation is in line with the recent studies of the mixed excitonic and CT states having strong interactions with the unharmonic environment; ${ }^{29}$ the second one can also be readily explained by this mechanism (see Extended Data Figure 8). The last one, in its turn, excludes the participation of the short-lived optically dark red-shifted fluorescence is related to the Chl-Chl interactions leading to the formation of a CT state (schematically shown in Figure 3d); the latter character of this state is also supported by the Stark fluorescence measurements. ${ }^{30}$

Most important is that our simulations also uncovered some properties of the quenched LHCII complexes that can help to distinguish between distinct mechanisms proposed so far to govern NPQ. ${ }^{3}$ First, contrarily to the case of the red-emitting complexes, the obtained mean concentration of the quenched complexes did not exhibit any notable endence on temperature ( $c f$. inset in Figure 3c), meaning that the physical origin of both states is different. This implies that quenching is unrelated to any CT states (either chlorophyll or carotenoid), in contrast to the earlier proposals. ${ }^{11}$ Indeed, if the quenched state were also related to a CT state, it should experience a considerable blue-shift upon cooling, resulting in a strong temperature dependence of the quenching efficiency that was not observed in our study. Second, our modelling results show that the quenched states of LHCIIs are energetically deep, with excitation de-trapping taking from hundreds of ps to tens of ns (see Figure $3 b$ ). This observation indicates a relatively fast unidirectional excitation transfer within the quenched complex from the Chl pigments to the intrinsic trapping centres and rules out the quenching mechanism based on the coherent mixing between the Chl and Car $\mathrm{S}_{1}$ states. ${ }^{13}$ Indeed, since the excitation flows between coherently coupled states more freely, it could also easily escape from the quenched complex itself back to the antenna, contradicting our determined minor reversibility of NPQ. As a result, the only remaining mechanism that is consistent with all our data is an incoherent excitation transfer to the short-lived $\mathrm{S}_{1}$ state of the Car molecules (Figure 3e), which also confirms that NPQ trap is rather slow. ${ }^{10}$ According to the structure-based quantum-chemical calculations, ${ }^{26}$ where the quenching efficiency of different carotenoids was assessed, the most probable candidate for the quenching site is one of the two luteins located in the centre of the LHCII monomer. Further experiments, for example, on LHCII mutants, are required to pinpoint the spatial location of the quenching site more accurately.

In conclusion, both the red-shifted fluorescence signal and fluorescence quenching observed in the LHCII aggregates were demonstrated to be the collective phenomena arising from the energetic interconnectivity of many conformationally fluctuating LHCII complexes, 
only several of them being in the red-emitting or quenched state. Moreover, all the known spectroscopic and structural similarities between the artificial LHCII aggregates and the intact thylakoids ${ }^{3}$ suggest that the molecular origin of the discussed conformational states should also remain the same during the in vivo photosynthesis, when about 6-10 LHCII trimers are closely inter-connected within the light-harvesting antenna of PSII. Indeed, recently developed fluctuating antenna model independently confirms that in both the LHCII aggregate and the thylakoid membranes (see Extended Data Figure 9 and Methods) excitation diffuses in a two-dimensional manner. Exposure to any ambient stress (like temperature, $\mathrm{pH}$ level, or even different levels of aggregation) shifts the dynamic equilibrium between the possible conformational states of a single LHCII (and possibly minor antenna complex), thus varying the relative concentrations of these states in the ensemble. As demonstrated in Figure $3 \mathrm{f}$, in small antenna systems just a slight variation of the concentration of the quenched complexes can significantly reduce the mean excitation lifetime and the resulting fluorescence quantum yield. The physical increase of the antenna size (indeed observed experimentally ${ }^{10}$ ) by merely a single LHCII trimer provides an additional possibility to enhance quenching efficiency. As a result, NPQ traps that can be (even with a very low probability of several percent) generated anywhere in the antenna allow photosynthetic organisms to gradually adapt to the varying light conditions and adjust NPQ efficiency by a certain degree that prevents photodamage.

Online Content Methods, along with any additional Extended Data display items, are available in the online version of the paper; references unique to these sections appear only in the online paper.

1. Croce, R. \& van Amerongen, H. Natural strategies for photosynthetic light harvesting. Nat. Chem. Biol. 10, 492-501 (2014).

2. van Amerongen, H., Valkunas, L. \& van Grondelle, R. Photosynthetic Excitons (World Scientific, Singapore, 2000).

3. Ruban, A. V., Johnson, M. P. \& Duffy, C. D. P. The photoprotective molecular switch in the photosystem II antenna. Biochim. Biophys. Acta, Bioenerg. 1817, 167-181 (2012).

4. Holt, N. E. et al. Carotenoid cation formation and the regulation of photosynthetic light harvesting. Science 307, 433-436 (2005).

5. Pascal, A. A. et al. Molecular basis of photoprotection and control of photosynthetic light-harvesting. Nature 436, 134-137 (2005).

6. Ruban, A. V. et al. Identification of a mechanism of photoprotective energy dissipation in higher plants. Nature 450, 575-578 (2007).

7. Ahn, T. K. et al. Architecture of a charge-transfer state regulating light harvesting in a plant antenna protein. Science 320, 794-797 (2008).

8. Staleva, H. et al. Mechanism of photoprotection in the cyanobacterial ancestor of plant antenna proteins. Nat. Chem. Biol. 11, 287-291 (2015).

9. Wientjes, E., van Amerongen, H. \& Croce, R. Quantum yield of charge separation in photosystem II: Functional effect of changes in the antenna size upon light acclimation. J. Phys. Chem. B 117, 11200-11208 (2013).

10. Belgio, E. et al. Economic photoprotection in photosystem II that retains a complete light-harvesting system with slow energy traps. Nat. Commun. 5, 4433 (2014).

11. Müller, M. G. et al. Singlet energy dissipation in the photosystem II light-harvesting complex does not involve energy transfer to carotenoids. ChemPhysChem 11, 1289-1296 (2010).

12. van Amerongen, H. \& van Grondelle, R. Understanding the energy transfer function of LHCII, the major light-harvesting complex of green plants. $J$. Phys. Chem. B 105, 604-617 (2001).

13. Walla, P. J., Linden, P. A., Ohta, K. \& Fleming, G. R. Excited-state kinetics of the carotenoid $S_{1}$ state in LHC II and two-photon excitation spectra of lutein and $\beta$-carotene in solution: Efficient $\mathrm{Car} \mathrm{S}_{1} \rightarrow \mathrm{Chl}$ electronic energy transfer via hot $S_{1}$ states? J. Phys. Chem. A 106, 1909-1916 (2002).

14. Horton, P. et al. Control of the light-harvesting function of chloroplast membranes by aggregation of the LHCII chlorophyll-protein complex. FEBS Lett. 292, 1-4 (1991).

15. Ruban, A. V., Young, A. \& Horton, P. Modulation of chlorophyll fluorescence quenching in isolated light harvesting complex of photosystem II. Biochim. Biophys. Acta, Bioenerg. 1186, 123-127 (1994).

16. Ruban, A. V., Dekker, J. P., Horton, P. \& van Grondelle, R. Temperature dependence of chlorophyll fluorescence from the light harvesting complex II of higher plants. Photochem. Photobiol. 61, 216-221 (1995).
17. Magdaong, N. M., Enriquez, M. M., LaFountain, A. M., Rafka, L. \& Frank, H. A. Effect of protein aggregation on the spectroscopic properties and excited state kinetics of the LHCII pigment-protein complex from green plants. Photosynth. Res. 118, 259-276 (2013).

18. Ware, M. A., Giovagnetti, V., Belgio, E. \& Ruban, A. V. PsbS protein modulates non-photochemical chlorophyll fluorescence quenching in membranes depleted of photosystems. J. Photochem. Photobiol. B 152, 301-307 (2015).

19. Krüger, T. P. J., Novoderezkhin, V. I., Ilioaia, C. \& van Grondelle, R. Fluorescence spectral dynamics of single LHCII trimers. Biophys. J. 98, 30933101 (2010).

20. Lawton, W. H. \& Sylvestre, E. A. Self modeling curve resolution. Technometrics 13, 617-633 (1971).

21. Chmeliov, J., Trinkunas, G., van Amerongen, H. \& Valkunas, L. Light harvesting in a fluctuating antenna. J. Am. Chem. Soc. 136, 8963-8972 (2014).

22. Schatz, G. H., Brock, H. \& Holzwarth, A. R. Picosecond kinetics of fluorescence and absorbance changes in photosystem II particles excited at low photon density. Proc. Natl. Acad. Sci. U. S. A. 84, 8414-8418 (1987).

23. Dekker, J. P. \& Boekema, E. J. Supramolecular organization of thylakoid membrane proteins in green plants. Biochim. Biophys. Acta, Bioenerg. 1706, 12-39 (2005).

24. Novoderezhkin, V. I., Palacios, M. A., van Amerongen, H. \& van Grondelle, R. Excitation dynamics in the LHCII complex of higher plants: Modeling based on the $2.72 \AA$ crystal structure. J. Phys. Chem. B 109, 10493-10504 (2005).

25. Müh, F., Madjet, M. E.-A. \& Renger, T. Structure-based identification of energy sinks in plant light-harvesting complex II. J. Phys. Chem. B 114, 13517-13535 (2010).

26. Chmeliov, J. et al. An 'all pigment' model of excitation quenching in LHCII. Phys. Chem. Chem. Phys. 17, 15857-15867 (2015).

27. Broess, K. et al. Excitation energy transfer and charge separation in photosystem II membranes revisited. Biophys. J. 91, 3776-3786 (2006).

28. Valkunas, L., Trinkunas, G., Chmeliov, J. \& Ruban, A. V. Modeling of exciton quenching in photosystem II. Phys. Chem. Chem. Phys. 11, 75767584 (2009).

29. Mančal, T., Valkunas, L. \& Fleming, G. R. Theory of exciton-charge transfer state coupled systems. Chem. Phys. Lett. 432, 301-305 (2006).

30. Wahadoszamen, M., Berera, R., Ara, A. M., Romero, E. \& van Grondelle, R. Identification of two emitting sites in the dissipative state of the major light harvesting antenna. Phys. Chem. Chem. Phys. 14, 759-766 (2012).

Supplementary Information is available in the online version of the paper.

Acknowledgements This work was supported by the Research Council of Lithuania (LMT grant no. MIP-080/2015). Computations were performed using the resources of the High Performance Computing Center "HPC Sauletekis" at Faculty of Physics, Vilnius University. A.V.R. would like to acknowledge the grants from The Leverhulme Trust and UK Biotechnology and Biological Sciences Research Council and The Royal Society for the Wolfson Research Merit Award. Authors also thank Erica Belgio and Petra Ungerer for the LHCII purification.

Author Contributions L.V. and A.V.R. designed the research, E.S. and R.A. performed the experiments, J.C. analysed experimental results in terms of selfmodelling curve resolution, described high-temperature fluorescence kinetics in terms of fluctuating antenna model, and made the figures of the manuscript, and A.G. modelled excitation energy transfer in LHCII aggregates. L.V., R.A., J.C., and A.G. contributed to the interpretation of the experimental results. J.C. and A.G. wrote the manuscript. L.V., R.A., A.V.R., and C.D.P.D. discussed and commented on the manuscript. 


\section{Methods}

\section{Sample preparations}

LHCII trimers were prepared from the BBY particles purified from the spinach leaves. Further purification of LHCII was done by sucrose density gradient centrifugation. Seven step exponential sucrose gradients from 0.15 to $1.0 \mathrm{M}$ sucrose dissolved in $15 \mathrm{mM}$ HEPES buffer with $0.03 \%(\mathrm{w} / \mathrm{v}) \mathrm{n}$-dodecyl $\beta$-D-maltoside $(\beta$-DM) were used. Sucrose was removed from LHCII using PD-10 desalting column (GE Healthcare) in a buffer containing $15 \mathrm{mM} \mathrm{HEPES}$ and $0.01 \%(\mathrm{w} / \mathrm{v}) \beta$ DM. Sample preparation technique is described in more details in literature. ${ }^{16,31,32}$ Aggregation of the sample was achieved with the help of bio-absorbent beads SM-2 (Bio-Rad). The amount of aggregation was observed as a drop of the fluorescence intensity at room temperature, when excited at $470 \mathrm{~nm}$. Sample quality was assessed by measuring it's steady-state absorption and fluorescence spectra. Proper aggregation level was considered achieved when the fluorescence intensity at $680 \mathrm{~nm}$ decreased tenfold. The aggregated solution then was diluted with glycerol, so that the final sample was $60 \%$ (v/v) glycerol solution. For the sake of consistency, all the data presented in the paper are obtained for the same batch of LHCII. Although we performed multiple additional measurements using LHCII from other three batches, we found no notable variations of fluorescence dynamics.

\section{Experimental measurements}

Time-resolved fluorescence dynamics of the samples were measured by means of Hamamatsu C5680 streak camera with either M5675 (synchroscan) or M5677 (single-sweep) module coupled to a spectrometer. Femtosecond Yb:KGW oscillator (Pharos, Light Conversion Ltd.) with a frequency doubler (HIRO, Light Conversion Ltd.) producing $515 \mathrm{~nm}$ sub-100-fs pulses at a $76 \mathrm{MHz}$ repetition rate was employed, and a pulse picker was used to reduce the repetition rate to $20 \mathrm{kHz}$ in the single-sweep mode for nanosecond timescales. The beam was attenuated down to about $1 \mathrm{pJ}$ ( $76 \mathrm{Mhz}$ repetition rate, picosecond timescales) or $100 \mathrm{pJ}$ ( $20 \mathrm{kHz}$ repetition rate, nanosecond timescales) per pulse and focused into about $100 \mu \mathrm{m}$ spot on the sample. No intensity dependence was observed when increasing the pulse energies by an order of magnitude. The temporal resolution of the whole system was $\sim 9.5 \mathrm{ps}$ (full width at half maximum (FWHM)) for the synchroscan mode and 90-110 ps for the single-sweep mode. All the measurements were performed in a fused silica cell of $0.1 \mathrm{~mm}$ optical path placed in a closed cycle helium cryostat (Janis Research Company, LLC). Steadystate absorption spectra were measured with Jasco V-670 absorption spectrometer. To avoid sample deterioration that may be caused by multiple freezing-thawing cycles, the cell was refilled after each cycle, thus, the long timescale and the high time-resolution fluorescence datasets and the absorption spectra are obtained during three separate experiments.

\section{Data analysis}

Often time-resolved fluorescence spectra are analysed in terms of the decay associated spectra (DAS). The observed non-exponential time dependence is then described by a number of states with distinct lifetimes (as an example, the three-exponential fit of our measured kinetics at 680 and $700 \mathrm{~nm}$ is shown in Table S1 in the Supplementary Information). The more exponentials are needed to describe the kinetics, the more intermediate states are obtained. While this type of analysis is suitable for small molecular systems, it is not the case for aggregates with constituents possessing different spectroscopic properties.
Indeed, in latter case the measured kinetics is an ensemble average of the kinetics with different timescales, coming from individual aggregates containing different numbers of distinct complexes. Therefore, analysis in terms of DAS could give intermediate states that have no physical origin.

Multivariate curve resolution. To avoid unnecessary intermediate states appearing in DAS analysis and to unambiguously distinguish between spectral components arising from the LHCII trimers being in different states, the collected time-resolved fluorescence spectra $F(\lambda, t)$ were analysed by implementing the multivariate curve resolution method. ${ }^{20}$ The main idea of this factorisation method is to express the initial data matrix as the product of two non-negative matrices, one for lineshapes of the distinct spectral components, $S_{i}(\lambda)$, and another for their relative time-dependent weights, $W_{i}(t)$ :

$$
F(\lambda, t) \approx \sum_{i=1}^{N} W_{i}(t) S_{i}(\lambda),
$$

or, in a matrix form,

$$
\mathbf{F}=\mathbf{W} \cdot \mathbf{S}+\mathbf{E},
$$

where the residual matrix $\mathbf{E}$ should be minimised. The number of the lineshapes $N$ should be kept minimal but sufficient to reproduce the experimental data-in our analysis, it was determined by means of the singular value decomposition that revealed two dominating singular values for the data collected at all the temperatures except the two highest ones. The factorisation was done according to the multiplicative update algorithm ${ }^{33}$ by using an additional unimodality constraint ensuring that each spectral component has only one dominating peak, thus minimising any possible ambiguity of the obtained results. All the optimisations were carried out for 10000 times starting from random non-negative initial matrices $\mathbf{W}$ and $\mathbf{S}$.

The fluorescence data collected for LHCII aggregates at the two highest temperatures, namely 250 and $273 \mathrm{~K}$, had only one dominating singular value indicating that only one spectral component should be sufficient. That was indeed supported by the multivariate curve resolution analysis that revealed fluorescence spectra having only single strong peak at $680 \mathrm{~nm}$, as shown in Extended Data Figure 4 (the dataset at $250 \mathrm{~K}$ was very similar to the one at $273 \mathrm{~K}$ and therefore is not shown). On the other hand, at lower temperatures the second component, having fluorescence maximum between 688 and $707 \mathrm{~nm}$, appears, see Extended Data Figures 3 and 4 as well as Figure 1 of the Manuscript. The best obtained results are summarised in Figure 2 of the Manuscript. The pure spectra $S_{i}(\lambda)$ were normalised to have equal areas under them. The obtained population kinetics of both components exhibit a clear non-exponential behaviour with the lifetimes varying from $\sim 200 \mathrm{ps}$ to $\sim 3 \mathrm{~ns}$.

The quality of the multivariate curve resolution analysis was validated by reconstructing the total fluorescence kinetics at various wavelengths and fluorescence spectra at different time delays ( $c f$. red lines in Extended Data Figures 3 and 4 as well as Figure 1 of the Manuscript). We clearly see a very good reproduction of the experimental timeresolved spectra during the initial 3 ns and somewhat worse results for the longer delay times. These discrepancies can arise due to several reasons. First of all, SMS measurements of the LHCII trimers have revealed occasional fluorescence at even redder spectrum region than described in our work (at 720-760 nm or even above $800 \mathrm{~nm}$ ), indicating the presence of more rarely accessible functional states of the light-harvesting complexes. ${ }^{19}$ The population of these states that are not accounted for in our spectral decomposition procedure, might have led to the mentioned discrepancies. Also, the amplitudes of the kinetics at later times drop substantially. Thus, the weight of the errors produced due to mis-fitting at the later time delays is actually very 
small. Indeed, the amplitude of the fluorescence spectrum, integrated over a 6-ns time interval (from 4 to $10 \mathrm{~ns}$ ) is smaller than that of the spectrum, integrated over a 0.3 -ns time interval from 1.2 to $1.5 \mathrm{~ns}$. In order to avoid any biasing in our following calculations, only the initial part (up to $3 \mathrm{~ns}$, where our decomposition is obviously valid) of the determined "pure" excitation kinetics were further analysed.

For reference, the same analysis was also performed on the solubilised non-aggregated LHCII trimers. Differently from the case of the aggregates, only one spectral component was sufficient to perfectly reproduce all the experimental data at all the temperatures (as an example, the $100 \mathrm{~K}$ dataset is shown in panels a-d of Extended Data Figure 1). This means that (i) the concentration of the red-emitting species of the LHCII trimers is relatively low and (ii) the intrinsic excitation decay rate in both $680-\mathrm{nm}$ and red species is very similar. The obtained fluorescence spectra and normalised kinetics at other temperatures are shown in Extended Data Figure 1e-f. Fluorescence spectra of the trimers were found to be very similar to those of the $680-\mathrm{nm}$ component in LHCII aggregates, $c f$. dots and solid lines in Figure 2a of the Manuscript, although some slight differences appear when the temperature drops below $\sim 130 \mathrm{~K}$. These discrepancies might be related to the state transitions experienced by the buffer solution at this temperature region. The fluorescence kinetics of LHCII trimers exhibited a mono-exponential decay behaviour and were further analysed by fitting them with the convolution of the instrument response function (IRF; approximated with a Gaussian function with FWHM of $110 \mathrm{ps}$ ) and a single-exponential function. From that fit, temperature-dependent fluorescence decay rates in LHCII trimers were obtained (see inset in Extended Data Figure 1f) and then used in the simulations of the excitation dynamics in the LHCII aggregate.

Finally, we applied our analysis procedure to the experimental results, obtained from the streak camera setup in the synchroscan mode with a much better time resolution resolution (FWHM of the IRF is just $9.5 \mathrm{ps}$ ) of the initial $125 \mathrm{ps}$ of the excitation decay kinetics in LHCII aggregates. Since at such a short time delay the population of the redemitting state is about an order of magnitude smaller than that of the dominating 680-nm-emitting state, we again were able to reproduce the whole fluorescence map, $F(\lambda, t)$ with just a single spectral component. The decomposed fluorescence spectra and normalised excitation kinetics are shown in Extended Data Figure 5. These kinetics were later used to further validate the results obtained from the modelling excitation dynamics in the LHCII aggregate.

Modelling excitation dynamics in LHCII aggregates. By summarising the results of our measured time-resolved fluorescence spectra (Figure 1 and Extended Data Figures 3 and 4), the performed multivariate curve resolution analysis (Figure 2) as well as single-molecule spectroscopy studies of single LHCII trimers, ${ }^{19}$ we draw several conclusions: (i) each LHCII monomer can be found in one of at least 3 distinct conformational states corresponding to the strong emission around $680 \mathrm{~nm}$, the red-shifted emission, or no emission at all; (ii) the switching between these states is a random process occurring on a micro- to millisecond timescale; (iii) excitation transfer in the aggregate is a diffusion-limited process. All these results can be summarised in the aggregate model presented in Figure 3a-b. Occasionally, all three monomers of the same LHCII trimer could even switch to three different states, as schematically shown in Figure 3a. This situation, however, is hardly observable during the SMS measurements as due to the fast inter-monomer excitation hopping rate all the excitation energy is quickly transferred to the quenched monomer. We note that recent SMS measurements ${ }^{34,35}$ and the corresponding theoretical modelling of the fluorescence blinking in single LHCII complexes ${ }^{36,37}$ have confirmed that the functional state of LHCII is most likely described by the conformation of its protein scaffold that deter- mines the inter-pigment couplings and excitation transfer rates. Due to trimeric structure of LHCII, the most favourable arrangement of the pigment-proteins within the aggregate should resemble a hexagonal lattice; such a composition of LHCII oligomers was indeed observed by means of electron microscopy. ${ }^{23}$ Of course, some deviations from such an arrangement are possible. Moreover, the peripheral complexes could even exhibit slightly different ability for switching between their functional states, compared to the complexes located deep inside the aggregate. Nonetheless, we do not expect these differences to be pronounced. Therefore, in order not to overcomplicate the model and to minimise the number of undefined parameters, such possibilities were not explicitly taken into account.

Structure-based calculations of the inter-pigment excitation dynamics in a LHCII monomer have revealed a relatively fast (of the order of several picoseconds) excitation transfer from chlorophyll $b$ to chlorophyll $a$ and subsequent equilibration. ${ }^{24-26,38}$ On the other hand, intercomplex excitation transfer rate is supposed to be about an order of magnitude slower, ${ }^{39}$ so that we can use a coarse-grained model ${ }^{27,28,40}$ to simulate excitation dynamics in the LHCII aggregate. We assume that LHCII monomer in each state can be described by a single value of (temperature-dependent) free energy $F(T)$, as shown in Figure 3b. Excitation hopping rates between the neighbouring complexes of the same free energy as well as those corresponding to a downward energy transfer are assumed to be the same and are denoted as $k_{\mathrm{h}}$. The rates of the energetically upward transitions are then rescaled by taking into account the Boltzmann factor. For example, the rate

$$
k_{\mathrm{red} \rightarrow 680}=k_{\mathrm{h}} \exp \left(-\frac{F_{680}-F_{\text {red }}}{k_{\mathrm{B}} T}\right),
$$

where $k_{\mathrm{B}}$ is Boltzmann constant and $T$ is an absolute temperature. The hopping rate $k_{\mathrm{h}}$ itself was assumed to similarly depend on temperature:

$$
k_{\mathrm{h}}=k_{\mathrm{h} 0} \exp \left(-\frac{\Delta E}{k_{\mathrm{B}} T}\right),
$$

where $\Delta E$ is some "activation energy" determining inter-complex excitation transfer that was evaluated by fitting fluorescence excitation kinetics. The parameter $k_{\mathrm{h} 0}$ was then chosen in such a way to ensure $k_{\mathrm{h}}^{-1}=25 \mathrm{ps}$ at room $(300 \mathrm{~K})$ temperature. ${ }^{21,39,41}$

Given all these possible excitation hopping rates between the adjacent monomers, we construct a hexagonal aggregate of 100 complexes, some of which are randomly chosen to be in the red-emitting state and some-in the quenched one. Assuming the homogeneous distribution of the initial excitation over the whole aggregate, its dynamics is calculated by solving the system of Pauli Master equations:

$$
\begin{aligned}
\frac{\mathrm{d}}{\mathrm{d} t} P_{i}(t) & =\sum_{j} k_{j \rightarrow i} P_{j}(t)-\sum_{j} k_{i \rightarrow j} P_{i}(t) \\
& -P_{i}(t) \times \begin{cases}k_{\mathrm{dis}}, & i \text { th complex is in the emitting state, } \\
k_{\text {trap },} & i \text { th complex is in the quenched state }\end{cases}
\end{aligned}
$$

where $P_{i}(t)$ is the population of the $i$ th complex, $k_{i \rightarrow j}$ is the intercomplex excitation hopping rate discussed above, $k_{\text {trap }}$ is the excitation trapping rate by the quenched LHCIIs, and $k_{\mathrm{dis}}=k_{\mathrm{fl}}+k_{\mathrm{nr}}$ is the total excitation dissipation rate due to fluorescence or non-radiative relaxation to the ground state. As discussed in the previous section, dissipation rate was assumed to be the same for both emitting states (the 680-nm and the red ones) and was determined from the time-resolved fluorescence measurements of non-aggregated LHCIIs (see inset in Extended Data Figure 1f for the temperature dependence of this dissipation rate). The total populations of the $680-\mathrm{nm}$ and red components are then calculated as the sum of the population of LHCIIs in a corresponding emitting state and afterwards are convolved with a Gaussian function 
(FWHM $=90 \mathrm{ps}$ ) representing the IRF of the experimental setup. The almost mono-exponential excitation decay kinetics, obtained in such a way, were then averaged over different random arrangements of the red and quenched LHCIIs within the aggregate as well as over various numbers of such complexes per aggregate. The latter were assumed to be distributed according to Poisson statistics:

$$
W_{N_{\text {red }}}^{\left\langle N_{\text {red }}\right\rangle}=\frac{\left\langle N_{\text {red }}\right\rangle^{N_{\text {red }}} \cdot \mathrm{e}^{-\left\langle N_{\text {red }}\right\rangle}}{N_{\text {red }} !}, \quad W_{N_{\mathrm{q}}}^{\left\langle N_{\mathrm{q}}\right\rangle}=\frac{\left\langle N_{\mathrm{q}}\right\rangle^{N_{\mathrm{q}}} \cdot \mathrm{e}^{-\left\langle N_{\mathrm{q}}\right\rangle}}{N_{\mathrm{q}} !},
$$

where $N_{\text {red }}$ and $N_{\mathrm{q}}$ are the actual numbers of the red and quenched complexes during the current realisation while $\left\langle N_{\text {red }}\right\rangle$ and $\left\langle N_{\mathrm{q}}\right\rangle$ denote the corresponding (temperature-dependent) mean values that describe the dynamic equilibrium of the conformational switching between the 3 possible states; $W_{N_{\text {red }}}^{\left\langle N_{\text {red }}\right\rangle}\left(W_{N_{\mathrm{q}}}^{\left\langle N_{\mathrm{q}}\right\rangle}\right)$ is the probability to have $N_{\text {red }}\left(N_{\mathrm{q}}\right)$ complexes in the aggregate. The maximum number of $N_{\text {red }}$ and $N_{\mathrm{q}}$ in Eq. 4 as well as the number of random arrangements considered in simulations were chosen to achieve converged kinetics. By taking into account these random fluctuations of the LHCII aggregate, we obtained the multi-exponential excitation decay kinetics that were then simultaneously fitted to the decomposed fluorescence kinetics of the two LHCII species shown in Figure 2c. All the fitting was done using the R software ${ }^{42}$ by utilising the differential evolution algorithm ${ }^{43}$ belonging to a class of genetic algorithms and implemented in Ref. 44. Fluorescence decay kinetics at different temperatures were fitted independently, thus allowing us to obtain the temperature dependence of the model parameters - the mean numbers of the red and quenched complexes as well as upward excitation transfer rates. For the latter, only rates $k_{\text {red } \rightarrow 680}$ and $k_{\mathrm{q} \rightarrow 680}$ were used as independent parameters, while transfer rates between the red and quenched complexes were chosen by setting the downward transfer rate to $k_{\mathrm{h}}$ and the upward transfer rate being calculated similarly to Eq. 1 . To minimise ambiguity of the fitting results, the excitation trapping rate $k_{\text {trap }}$ was fixed to be the same at all the temperatures. Moreover, the determined relative amplitudes of the 680-nm and red component kinetics ( $c f$. Figure 1e) were also taken into account, thus additionally restricting possible ranges for the $\left\langle N_{\text {red }}\right\rangle$ values. All the kinetics were calculated up to $3 \mathrm{~ns}$, where our decomposition is valid ( $c f$. Extended Data Figures 3 and 4) and the population of any additional states (possibly more pronounced at later times) can be neglected.

We would like to emphasise at this point that our model is the simplest possible that allowed us to simultaneously fit all the experimental data collected at all the considered temperatures. First, we used the minimal number (three) of functional states as a two-state model was found to be inadequate ( $c f$. Figure $\mathrm{S} 1$ in the Supplementary Information). The arrangement of the complexes in a hexagonal lattice is the most natural for trimeric LHCIIs. The total number of complexes, 100, was chosen to represent large but not yet infinite aggregate, and was not varied during the fits. The distribution of both the red-emitting and quenched complexes was totally random, which is again the simplest assumption that prevents us from postulating any uncertain clustering or peripheral effects. While the mean numbers of the red-emitting and the quenched complexes were treated as free parameters, their exact numbers at each specific aggregate realisation were chosen according to the commonly used Poisson statistics. The hopping rate between the same kind of complexes should obviously depend on temperature. Nonetheless, we avoided having it as free parameter for all the temperatures and instead used a simple exponential law (Eq. 2), which, after choosing the hopping time $k_{\mathrm{h}}^{-1}=25 \mathrm{ps}$ at room temperature, leaves only one free parameter, $\Delta E$, to determine those at other temperatures. We further made an additional simplification that energetically downward hopping rate between the adjacent complexes in different conformational states is the same as between the complexes of the same kind.
Additional two free parameters were energetically upward transition rates from the red-emitting and the quenched complexes to the 680-nm ones. Note that despite a simple form of the Boltzmann factor in Eq. 1, it cannot be described by a single parameter for all the temperatures, as the free energy $F(T)$ of the complex in general is a complicated temperature-dependent function that could be simplified only if all the pigments within the LHCII complex had the same excited state energy, which is obviously not the case. ${ }^{24,25}$ Meanwhile, to describe the hopping rates between the red-emitting and the quenched complexes, no additional free parameters were necessary, as discussed above. Furthermore, the dissipation rate of both the $680-\mathrm{nm}$ and the red-emitting complexes were postulated to be the same, as this simple assumption was supported by our fluorescence measurements in the LHCII trimers. The trapping rate of the quenched complexes was the final free parameter in our model. Nevertheless, we made a simplification and set its value to be the same at all the considered temperatures. In summary, we stress that our model is the simplest possible three-state model of the LHCII aggregate with the minimal number of free parameters.

During our simulations, we have tried different $\Delta E$ values appearing in Eq. 2 and found that the best results were obtained for $\Delta E=18 \mathrm{~cm}^{-1}$ (though similar quality of the fit can be achieved for $\Delta E$ being between 16 and $\left.22 \mathrm{~cm}^{-1}\right)$. After choosing $\Delta E$, we have set $k_{\text {trap }}$ to $(100 \mathrm{ps})^{-1},(75 \mathrm{ps})^{-1},(50 \mathrm{ps})^{-1},(25 \mathrm{ps})^{-1}$ or $\infty$ (corresponding to infinitely fast/infinitely deep trap). The last one was found to give somewhat worse results than the finite values. For all non-infinite trapping rates, the resulting fit was of a similar quality and the obtained values for $\left\langle N_{\mathrm{q}}\right\rangle,\left\langle N_{\text {red }}\right\rangle$ and $k_{\text {red } \rightarrow 680}$ showed the same qualitative temperature dependence and converged to roughly the same values ( $c f$. Extended Data Figure $6 \mathrm{~b}-\mathrm{d})$. The de-trapping rate $k_{\mathrm{q} \rightarrow 680}$, however, exhibited large scatter. Nonetheless, its values in all cases varied from $(500 \mathrm{ps})^{-1}$ to $(10 \mathrm{~ns})^{-1}$.

For two highest temperatures, $250 \mathrm{~K}$ and $273 \mathrm{~K}$, we had to make additional simplifications, because our decomposition provided the kinetics of just a single component. Therefore, we assumed that the number of complexes in the red state is vanishingly small (this assumption completely agrees with overall temperature dependence of concentration of the red complexes, see Extended Data Figure 6d).

The obtained fitting results for $\Delta E=18 \mathrm{~cm}^{-1}$ and various $k_{\text {trap }}$ values are presented in Extended Data Figure 6a. We see that at all temperatures, our model can reasonably reproduce the excitation decay kinetics of both emitting states (the 680-nm and the red one) as well as the relative amplitudes of both kinetics. Some discrepancies with the initial rise part of the kinetics from the red complexes can be noted, but they can be attributed to the mis-fitting of the multivariate curve resolution procedure at this region of the strong domination of the 680-nm component; nevertheless, the maximum of the kinetics of red component was captured by our model at the correctly delayed position.

As already mentioned, the obtained parameters for all the considered $k_{\text {trap }}$ values are shown in Extended Data Figure $6 \mathrm{~b}-\mathrm{d}$. The temperature dependence of the hopping time for $\Delta E=18 \mathrm{~cm}^{-1}$ is shown with the black line in Extended Data Figure 6b. The back-transfer time from the red to the 680 -nm emitting complex was evaluated to vary between $\sim 800 \mathrm{ps}$ at $200 \mathrm{~K}$ to several $\mathrm{ns}$ at lower temperature, indicating the relative "energetic deepness" of the red state. All these excitation transfer times are also summarised in Figure 3b. Finally, the obtained mean numbers of the complexes in the red and quenched states are presented in Extended Data Figure 6c and d, respectively. While the latter does not exhibit any notable variations and only slightly fluctuates around the value $\left\langle N_{\mathrm{q}}\right\rangle \approx 8$ (indicated with the dashed black line), the former drops almost by an order of magnitude upon temperature increase from 15 to $200 \mathrm{~K}$. At higher temperatures, our obtained concentrations are consistent with the results of the single-molecule spectroscopic measurements of single LHCII trimers. ${ }^{19}$ 
If the $\Delta E$ value were somewhat different, the absolute values of the obtained parameters would also change (mostly at low temperature, see Figure S2 in the Supplementary Information). Nonetheless, the qualitative behaviour, upon which all our conclusions are based, remains the same. That is, the mean number of red complexes always shows huge increase with decreasing temperature, while the number of quenched complexes remains roughly constant. Also, in no case the de-trapping rate from the quenched state was faster than $(300 \mathrm{ps})^{-1}$. Thus, our conclusions about the molecular origin of the red and quenched states hold anyway.

To further validate our modelling results, we also calculated the convolution of the simulated excitation decay kinetics with the much shorter IRF (FWHM $=9.5 \mathrm{ps}$ ) and compared them with the kinetics measured in the single-sweep mode. The simulated kinetics follow the experimental ones pretty well at all the temperatures, as shown in Extended Data Figure 7. That provides an additional support for our model of LHCII aggregate, being as simple as possible but still capturing all the major spectroscopic and time-resolved properties of the excitation energy transfer, thus strengthening our main conclusions discussed in the Manuscript.

All the results presented so far are related either to the individual LHCII trimers or to their large aggregate. In thylakoid membranes, however, LHCIIs are found to form moderately sized clusters, about 3-4 LHCII trimers per RC. ${ }^{2}$ Thus the pool of the light-harvesting complexes, separated by the RCs of different PSIIs is composed on average of 6-10 LHCII trimers. To evaluate quenching efficiency in such small antenna systems, we calculated the mean excitation lifetime as a function of the aggregate size and the mean concentration of the quenched complexes within the aggregate:

$$
\langle\tau\rangle=\int_{0}^{\infty} P(t) \mathrm{d} t,
$$

where $P(t)$ is the total time-dependent population of the $680-\mathrm{nm}$ emitting complexes, normalised to 1 at $t=0$. For these calculations, we used the parameters extracted from our analysis presented above and corresponding to $273 \mathrm{~K}$, the highest temperature for which we have $k_{\text {dis }}$ values in LHCII trimers. Particularly, we used $\Delta E=18 \mathrm{~cm}^{-1}$, $k_{\text {trap }}^{-1}=50 \mathrm{ps}$, and $k_{\mathrm{q} \rightarrow 680}^{-1}=2 \mathrm{~ns}$ (the calculations were not sensitive to the variations of the last one). For simplicity, no red-emitting complexes were included in this analysis since our above calculations suggest vanishingly small concentration of the red-emitting states at higher temperatures. By changing the size of the aggregate and the mean number of LHCII monomers being in the quenched state, the obtained variations in the mean excitation lifetime are presented in Figure $3 f$. We would like to mention at this point that neither different mutual arrangement of the light-harvesting complexes nor possible variations of the inter-complex excitation hopping time, possibly occurring in the in vivo thylakoid membranes, would result in any notable change to this dependency (see Figure S3 in the Supplementary Information). As a result, the probability of just several percent for LHCII to switch into its quenched state is enough to reduce the mean fluorescence lifetime from $\sim 4$ ns seen in LHCII trimers down to $\sim 2$ ns observed in intact thylakoids with the closed $\mathrm{RCs}^{45}$ or even smaller values in LHCII liposomes. ${ }^{46}$ Further environmentally induced slight increase of the concentration of the quenched complexes, potentially accompanied with a physical increase of the antenna size, ${ }^{10}$ can provide additional 3-4-fold drop of the fluorescence quantum yield, typically observable under NPQ conditions.

Fluctuating antenna model. To verify our conclusions regarding the obtained mean concentration of the quenched complexes and to further support the postulated two-dimensional arrangement of the LHCII complexes within the aggregate, we have also analysed the fluorescence decay kinetics from Figure 2c, corresponding to the $273 \mathrm{~K}$ temperature, in terms of completely different approach. As discussed above, the multi-exponential fluorescence decay kinetics in the LHCII aggregate can be reproduced only by averaging them over the random distribution of the quenched (and red-emitting) complexes within the aggregate as well as over their exact number per aggregate. However, such an explanation can not hold for the purified photosystems II, where the position of the excitation traps - the reaction centres - is strongly defined while the fluorescence kinetics still exhibits multi-exponential decay behaviour independently of the antenna size. ${ }^{47}$ For several decades, this decay behaviour was attributed to the reversible charge separation in the RCs, but it has been proposed recently that its origin might also be related to the fluctuating properties of the light-harvesting antenna itself, like varying inter-complex connectivity. As a result, a fluctuating antenna model dealing with a simple excitation diffusion in a continuous medium was formulated and then successfully applied to the very different photosynthetic light-harvesting systems, ranging from PSIIs of different antenna size or LHCII aggregates to the BBY preparations or even the whole thylakoid membranes. ${ }^{21,48}$ In all the cases, the multiexponential fluorescence kinetics was reproduced using just 3 parameters, one of them being the fractal dimensionality $d$ of the considered systems, the second one, $\eta$, relating the mean excitation diffusion rate to the mean concentration of the traps per system, and, finally, the intrinsic dissipation rate $k_{\text {dis }}$.

As was already discussed above, at high temperature the redemitting states can be neglected, which allows us to apply this fluctuating antenna model to our obtained fluorescence kinetics at $273 \mathrm{~K}$. By convolving the calculated excitation population kinetics with the IRF (represented by the Gaussian function with FWHM of $90 \mathrm{ps}$ ), the obtained kinetics was fitted to the experimental data using the ordinary least-squares-based algorithm. As in our coarse-grained modelling described above, during these calculations the intrinsic decay rate was taken from the inset of Figure if and therefore was fixed to be $k_{\mathrm{dis}}^{-1}=4.2 \mathrm{~ns}$. The calculated best-fitted kinetics together with the obtained remaining two model parameters $(d$ and $\eta)$ are presented in Extended Data Figure 9a. First of all, we note that, similarly to the previously studied PSII supercomplexes, ${ }^{21,48}$ the obtained dimensionality is $d \approx 1.7$, which reveals the existence of some distortions in the planar distribution and/or connectivity of the pigment-protein complexes within the aggregate. In our previous study, the dimensionality $d \approx 1.9<2$ was obtained even for the thylakoid membranes, ${ }^{48}$ which indicates the absence of any (3-dimensional) inter-layer excitation transfer under in vivo conditions. This result further supports the two-dimensional arrangement of the LHCII complexes within the aggregate that we postulated during our coarse-grained modelling and also emphasises another common property (in addition to those listed in the introduction) between the in vitro LHCII aggregates and in vivo photosynthetic systems.

Another obtained model parameter, $\eta=1.3 \mathrm{~ns}$, provides information about the concentration of the traps per aggregate. It can be related to the parameters of the coarse-grained model as ${ }^{21,48}$

$$
\eta \simeq 2 d k_{\mathrm{h}}^{-1}\left(\left\langle N_{\mathrm{q}}\right\rangle / N\right)^{-2 / d}
$$

where $k_{\mathrm{h}}$ is the mean inter-complex excitation hopping time and $\left\langle N_{\mathrm{q}}\right\rangle / N$ is the relative number of the quenched complexes per aggregate. The relationship between $k_{\mathrm{h}}^{-1}$ and $\left\langle N_{\mathrm{q}}\right\rangle / N$, given by Eq. 6 , is demonstrated in Extended Data Figure $9 \mathrm{~b}$. Interestingly, if we choose $k_{\mathrm{h}}^{-1}=25 \mathrm{ps}$ and $N=100$, we can approximate $\left\langle N_{\mathrm{q}}\right\rangle \approx 10$ (as indicated with a star in Extended Data Figure $9 \mathrm{~b}$ ), which is similar to the value $\left\langle N_{\mathrm{q}}\right\rangle \approx 8$ attained from our coarse-grained model ( $c$. Extended Data Figure 6d). These comparable results, obtained from two completely different and independent models, further support the conclusions of our work. 
Code availability. Program codes are available upon request to the corresponding author.

31. Ruban, A. V., Young, A. J., Pascal, A. A. \& Horton, P. The effects of illumination on the xanthophyll composition of the photosystem II lightharvesting complexes of spinach thylakoid membranes. Plant Physiol. 104, 227-234 (1994).

32. Rutkauskas, D., Chmeliov, J., Johnson, M., Ruban, A. \& Valkunas, L. Exciton annihilation as a probe of the light-harvesting antenna transition into the photoprotective mode. Chem. Phys. 404, 123-128 (2012).

33. Berry, M. W., Browne, M., Langville, A. N., Pauca, V. P. \& Plemmons, R. J. Algorithms and applications for approximate nonnegative matrix factorization. Comput. Stat. Data Anal. 52, 155-173 (2007).

34. Krüger, T. P. J., Ilioaia, C. \& van Grondelle, R. Fluorescence intermittency from the main plant light-harvesting complex: Resolving shifts between intensity levels. J. Phys. Chem. B 115, 5071-5082 (2011).

35. Krüger, T. P. J., Ilioaia, C., Valkunas, L. \& van Grondelle, R. Fluorescence intermittency from the main plant light-harvesting complex: Sensitivity to the local environment. J. Phys. Chem. B 115, 5083-5095 (2011).

36. Valkunas, L., Chmeliov, J., Krüger, T. P. J., Ilioaia, C. \& van Grondelle, R. How photosynthetic proteins switch. J. Phys. Chem. Lett. 3, 2779-2784 (2012).

37. Chmeliov, J., Valkunas, L., Krüger, T. P. J., Ilioaia, C. \& van Grondelle, R Fluorescence blinking of single major light-harvesting complexes. New J. Phys. 15, 085007 (2013).

38. Duffy, C. D. P. et al. Modeling of fluorescence quenching by lutein in the plant light-harvesting complex LHCII. J. Phys. Chem. B 117, 10974-10986 (2013).

39. Bennett, D. I. G., Amarnath, K. \& Fleming, G. R. A structure-based model of energy transfer reveals the principles of light harvesting in photosystem II supercomplexes. J. Am. Chem. Soc. 135, 9164-9173 (2013).

40. Valkunas, L. et al. Excitation migration, quenching, and regulation of photosynthetic light harvesting in photosystem II. J. Phys. Chem. B 115, 92529260 (2011).

41. Barzda, V. et al. Singlet-singlet annihilation kinetics in aggregates and trimers of LHCII. Biophys. J. 80, 2409-2421 (2001).

42. R Core Team. R: A Language and Environment for Statistical Computing. R Foundation for Statistical Computing, Vienna, Austria (2013). URL http: //www.R-project.org/.

43. Price, K. V., Storn, R. M. \& Lampinen, J. A. Differential Evolution. A Practical Approach to Global Optimization. Natural Computing Series (Springer-Verlag Berlin Heidelberg, 2005).

44. Ardia, D., Mullen, K. M., Peterson, B. G. \& Ulrich, J. DEoptim: Differential Evolution in R (2015). URL http://CRAN.R-project.org/ package=DEoptim. Version 2.2-3.

45. Belgio, E., Johnson, M. P., Jurić, S. \& Ruban, A. V. Higher plant photosystem II light-harvesting antenna, not the reaction center, determines the excited-state lifetime- both the maximum and the nonphotochemically quenched. Biophys. J. 102, 2761-2771 (2012).

46. Moya, I., Silvestri, M., Vallon, O., Cinque, G. \& Bassi, R. Time-resolved fluorescence analysis of the photosystem II antenna proteins in detergent micelles and liposomes. Biochemistry 40, 12552-12561 (2001).

47. Caffarri, S., Broess, K., Croce, R. \& van Amerongen, H. Excitation en ergy transfer and trapping in higher plant photosystem II complexes with different antenna sizes. Biophys. J. 100, 2094-2103 (2011).

48. Chmeliov, J., Trinkunas, G., van Amerongen, H. \& Valkunas, L. Excitation migration in fluctuating light-harvesting antenna systems. Photosynth. Res. 127, 49-60 (2016). 

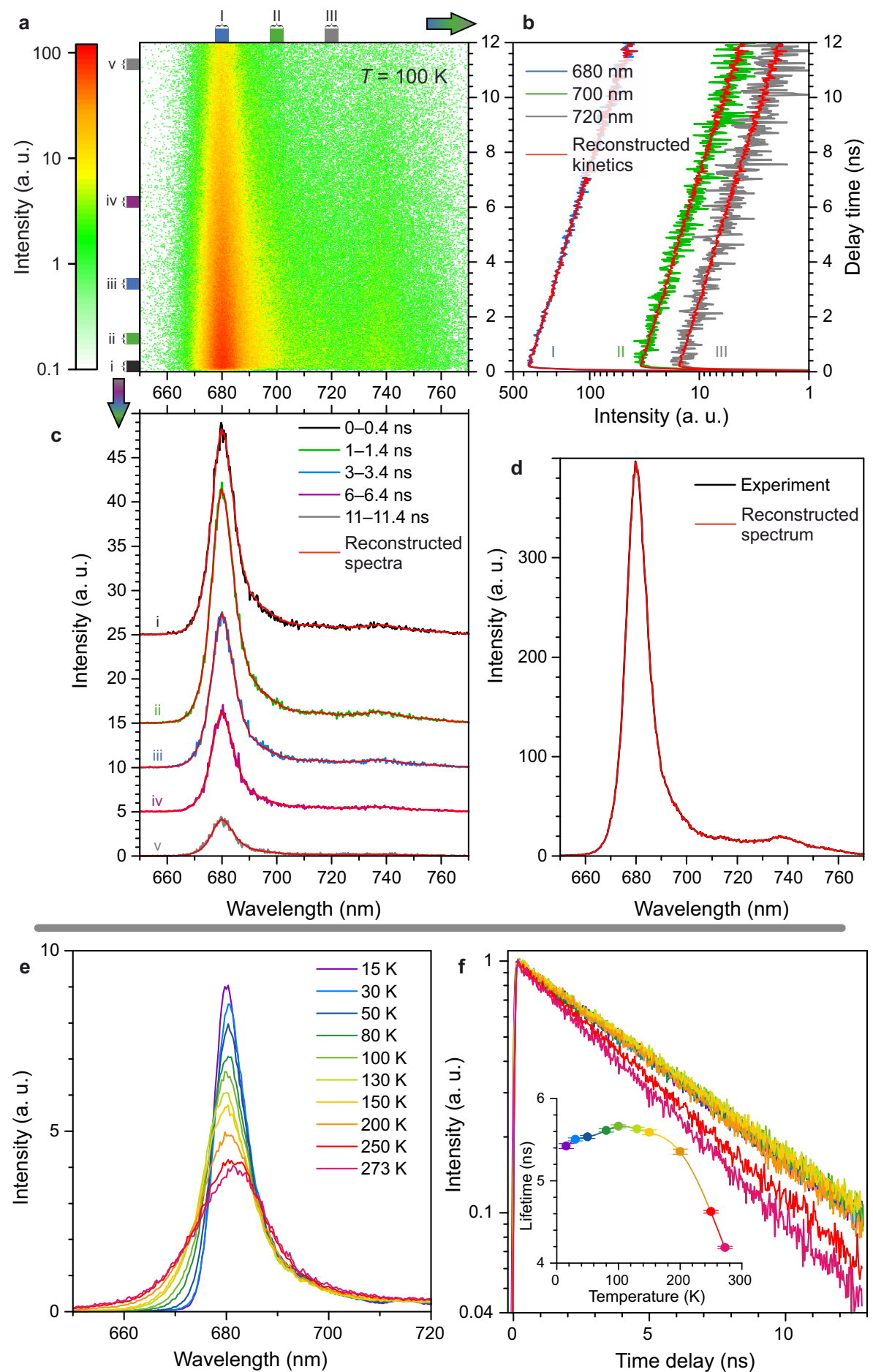

Extended Data Figure 1 । Results of the multivariate curve resolution analysis, performed on the time-resolved fluorescence spectra of LHCII trimers. a, Two-dimensional plot illustrating evolution of fluorescence intensity $F(\lambda, t)$ at $100 \mathrm{~K}$ temperature. b, vertical slices (fluorescence kinetics) of fluorescence map in panel (a), obtained by integrating it over a 5-nm-wide regions around 680, 700, and $720 \mathrm{~nm}$ (indicated with colour bars I-III). c, horizontal slices (fluorescence spectra) of fluorescence map in panel (a), obtained by integrating it over different time intervals (indicated with colour bars $\mathrm{i}-\mathrm{v}$ ). d, total fluorescence spectrum of LHCII trimers, obtained by integrating fluorescence intensity map in panel (a) over the whole time region. Red lines in panels (b-d) illustrate corresponding fluorescence kinetics and spectra, reconstructed from the multivariate curve resolution analysis by using just a single spectral component, see Manuscript text and Methods for details. Similar results were obtained for other temperatures, too, as summarised in panels (e-f). e, obtained fluorescence spectra at various temperatures, normalised to have equal area below them. f, obtained normalised fluorescence kinetics at those temperatures. Inset, mean excitation lifetimes in LHCII trimers at various temperatures, determined from the single-exponential reconvolution fit of the fluorescence kinetics shown in panel (f). Horizontal lines present standard deviations for the corresponding lifetimes. 

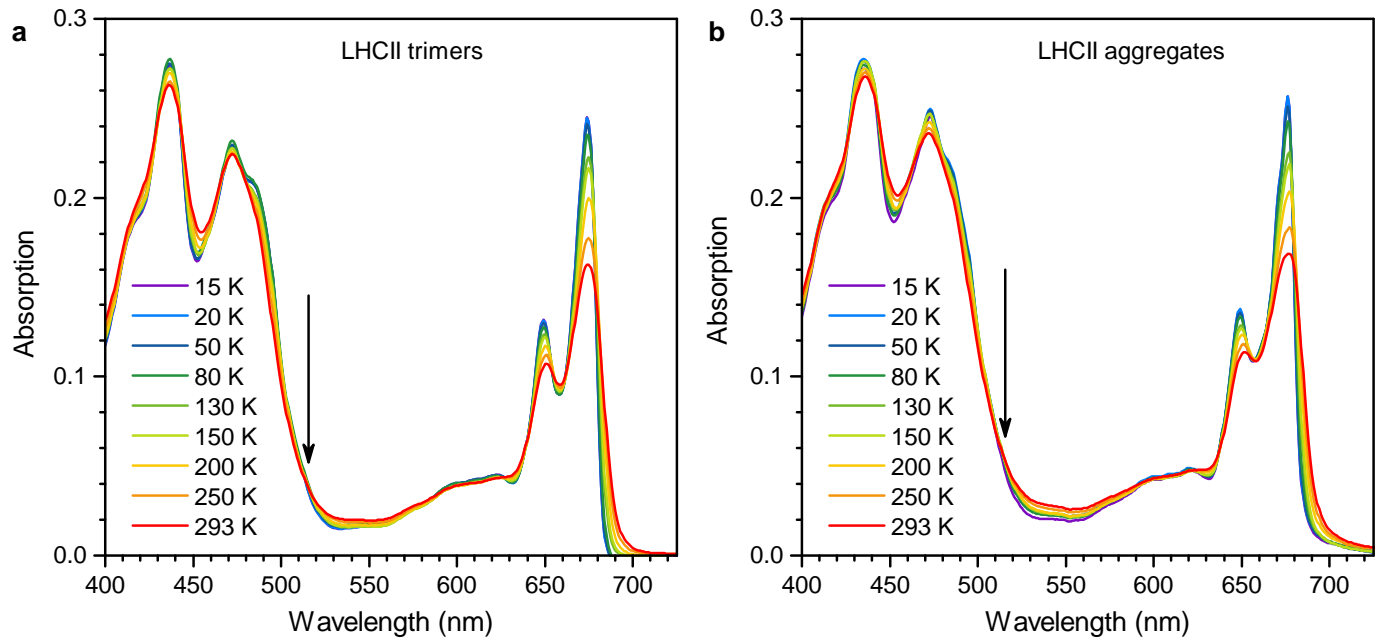

Extended Data Figure 2 I Absorption spectra of LHCII trimers (a) and aggregates (b) at different temperatures. Arrows denote the excitation wavelength $(515 \mathrm{~nm})$ used in our time-resolved fluorescence measurements. 

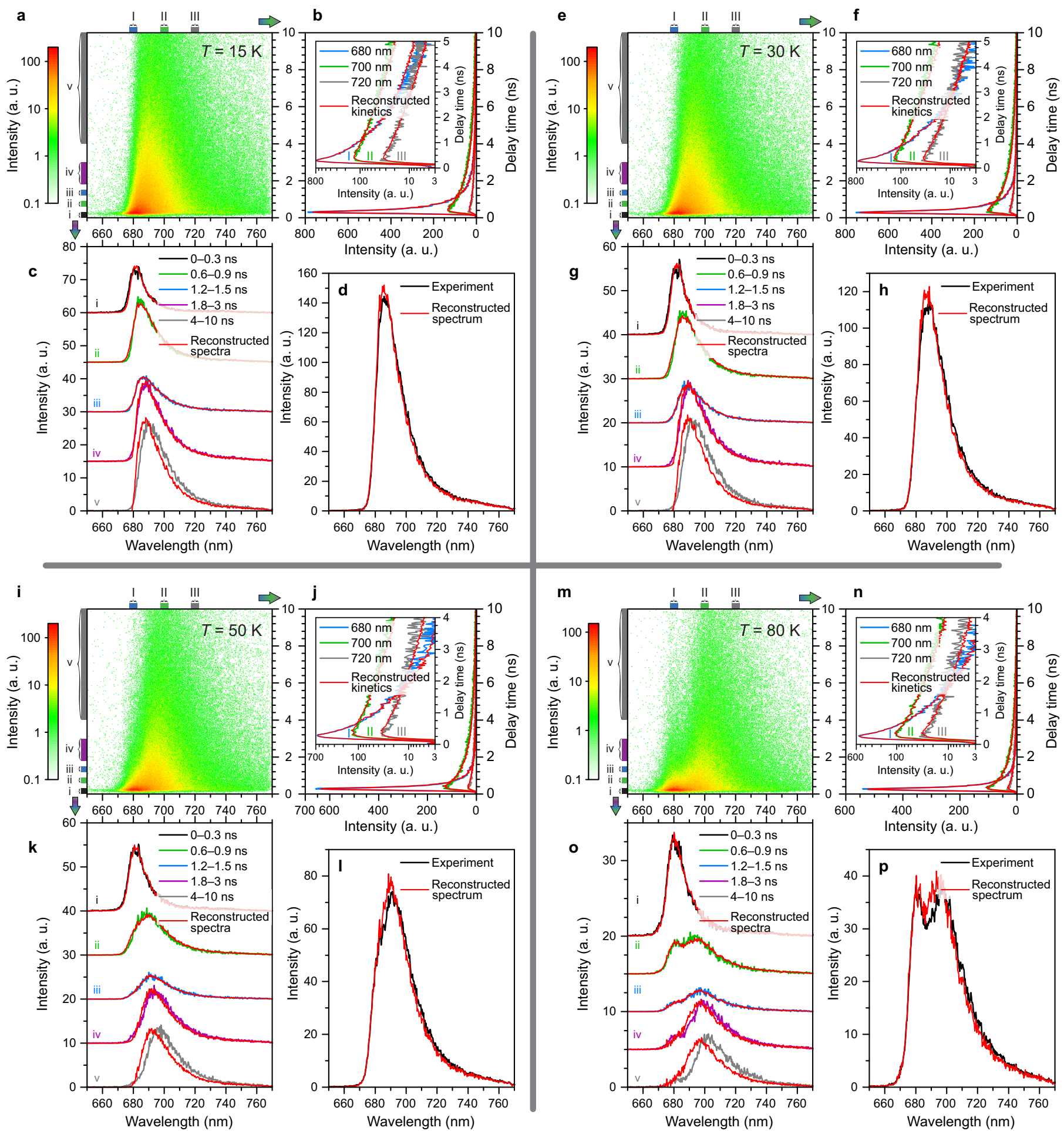

Extended Data Figure 3 । Time-resolved fluorescence measurements of LHCII aggregates at 15 K (a-d), 30 K (e-h), 50 K (i-l), and $80 \mathrm{~K}(\mathbf{m}-\mathbf{p})$ temperatures. Top-left, two-dimensional plot illustrating evolution of fluorescence intensity $F(\lambda, t)$ at particular temperature. Top-right, vertical slices (fluorescence kinetics) of fluorescence map obtained by integrating it over a 5 -nm-wide regions around 680, 700, and $720 \mathrm{~nm}$ (indicated with colour bars I-III). Inset, the same kinetics on a semi-logarithmic scale. Bottom-left, horizontal slices (fluorescence spectra) of fluorescence map obtained by integrating it over different time intervals (indicated with colour bars i-v). Bottom-right, steady-state fluorescence spectrum of LHCII aggregate, obtained by integrating fluorescence intensity map over the whole time region. Red lines in all panels illustrate corresponding fluorescence kinetics and spectra, reconstructed from the multivariate curve resolution analysis, see Manuscript text and Methods for details. 

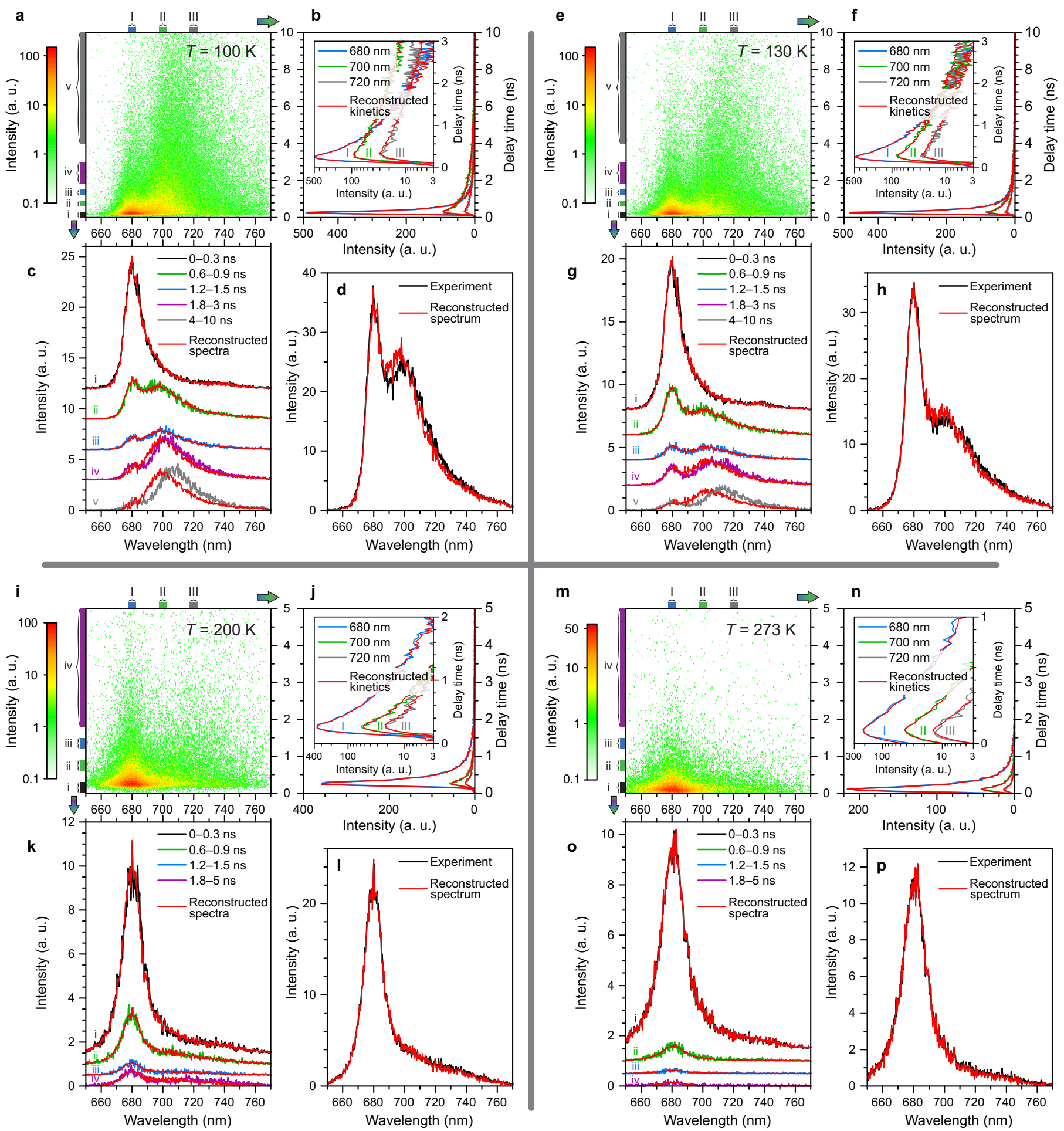

Extended Data Figure 4 I Time-resolved fluorescence measurements of LHCII aggregates at $100 \mathrm{~K}$ (a-d), $130 \mathrm{~K}$ (e-h), $200 \mathrm{~K}$ (i-l), and $273 \mathrm{~K}(\mathbf{m}-\mathbf{p})$ temperatures. Top-left, two-dimensional plot illustrating evolution of fluorescence intensity $F(\lambda, t)$ at particular temperature. Top-right, vertical slices (fluorescence kinetics) of fluorescence map obtained by integrating it over a 5-nm-wide regions around 680, 700, and $720 \mathrm{~nm}$ (indicated with colour bars I-III). Inset, the same kinetics on a semi-logarithmic scale. Bottom-left, horizontal slices (fluorescence spectra) of fluorescence map obtained by integrating it over different time intervals (indicated with colour bars i-v). Bottom-right, steady-state fluorescence spectrum of LHCII aggregate, obtained by integrating fluorescence intensity map over the whole time region. Red lines in all panels illustrate corresponding fluorescence kinetics and spectra, reconstructed from the multivariate curve resolution analysis, see Manuscript text and Methods for details. 

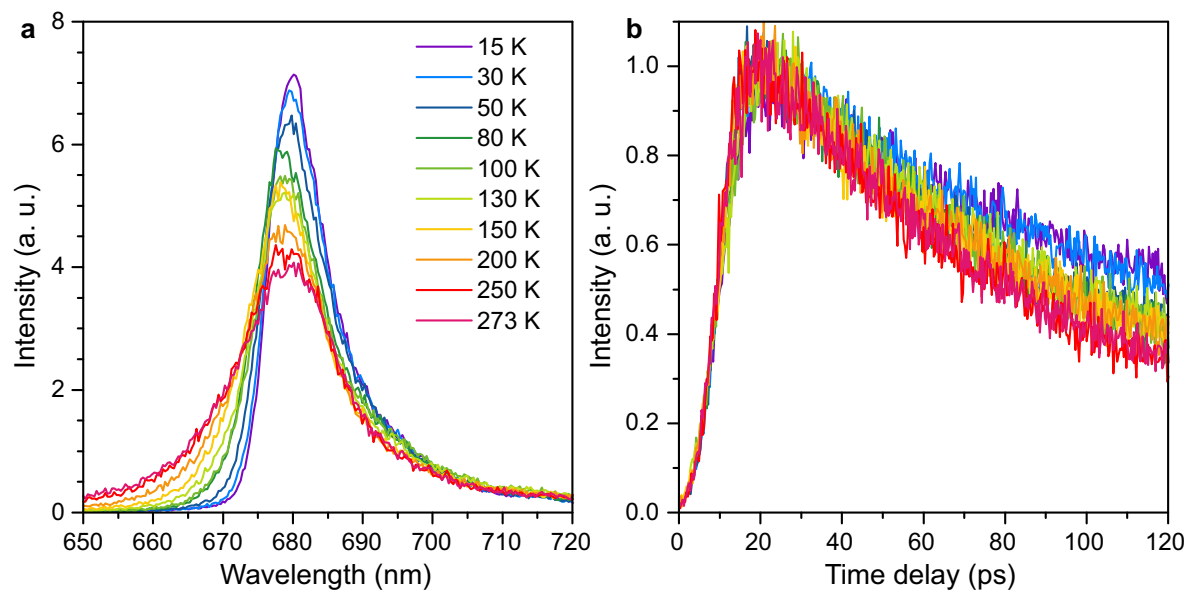

Extended Data Figure 5 I Results of the multivariate curve resolution analysis, performed on the high-resolution time-resolved fluorescence spectra of LHCII aggregates, measured in synchroscan mode. a, obtained fluorescence spectra at various temperatures, normalised to have equal area below them. b, obtained normalised fluorescence kinetics at those temperatures. 

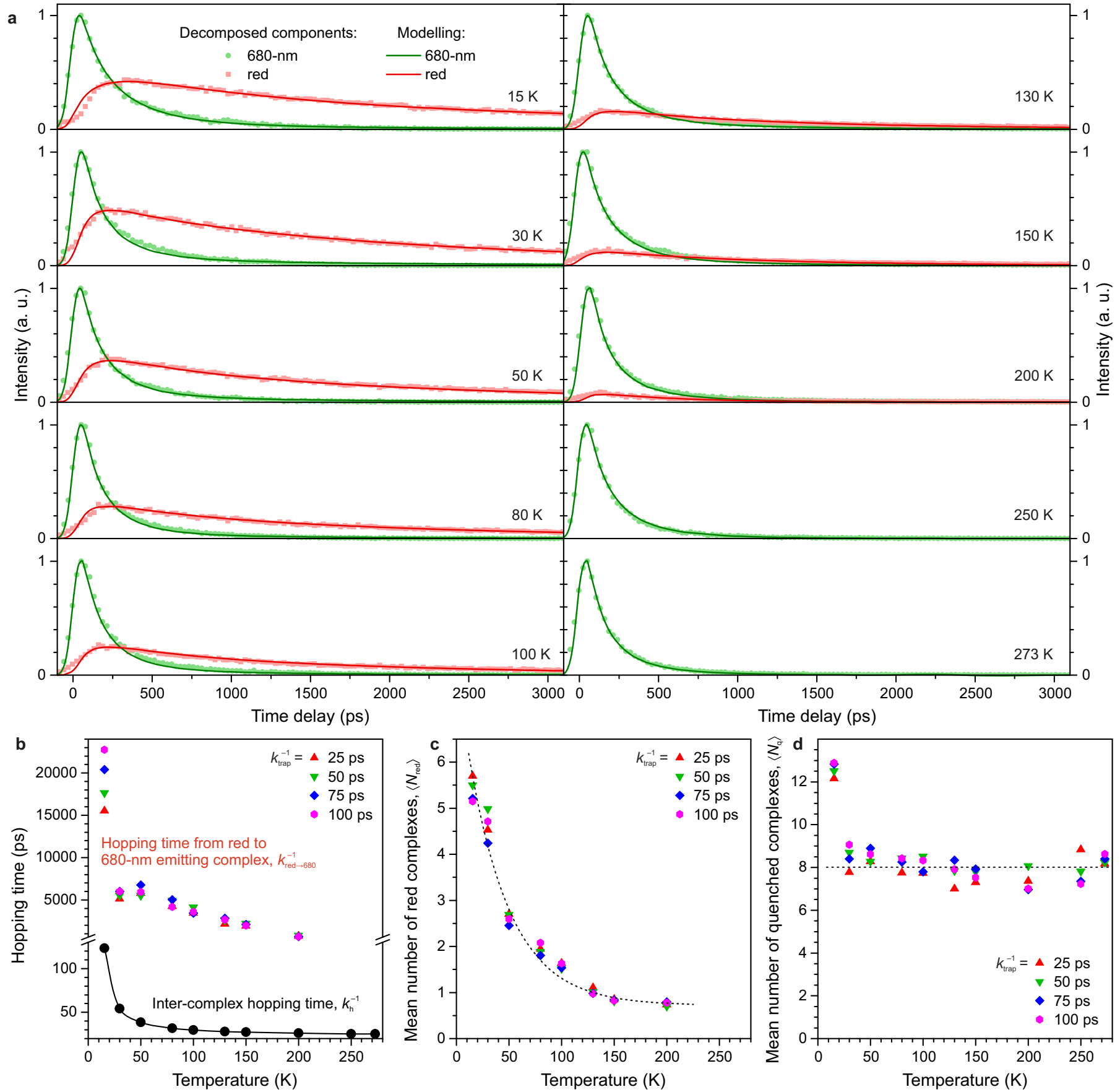

Extended Data Figure 6 I Results of the modelling fluorescence kinetics in LHCII aggregate. a, fitted fluorescence kinetics of the 680-nm and red components in LHCII aggregate at various temperatures. b, inter-complex excitation hopping times, determined by fitting fluorescence kinetics in the panel (a), see Figure 3a-b for definition. Black line represent the temperature dependence given by Eq. 2. c and d, obtained mean numbers of the complexes being in the red-emitting (c) and quenched (d) states. 


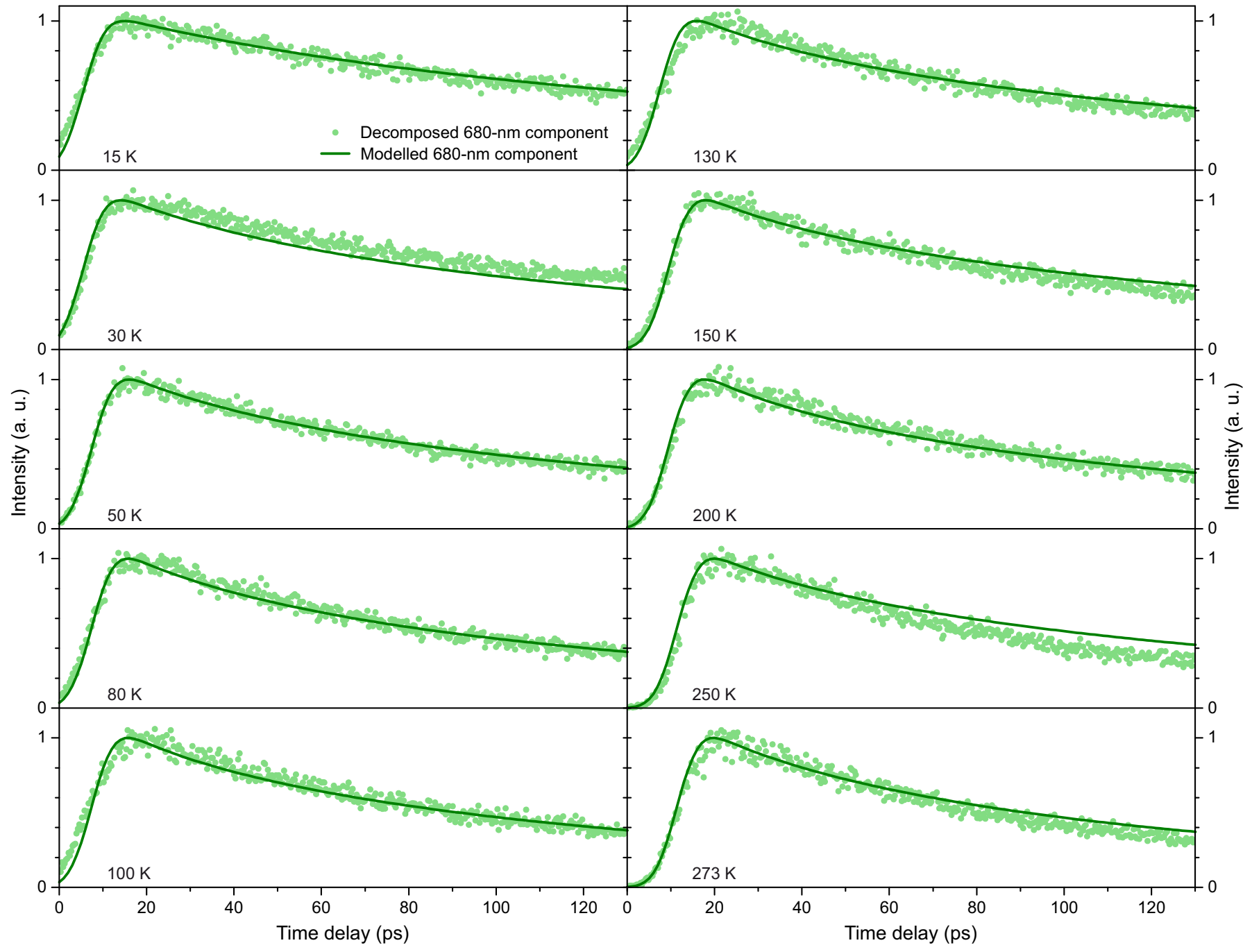

Extended Data Figure 7 I Simulations of the initial part of the fluorescence decay kinetics in LHCII aggregates. 
High temperature

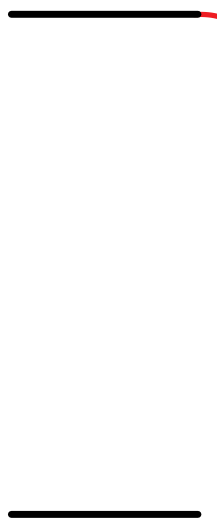

Chl
Chl-Chl CT states

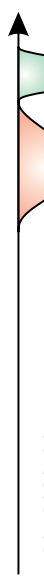

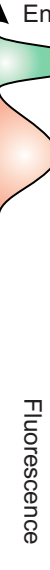

Low temperature

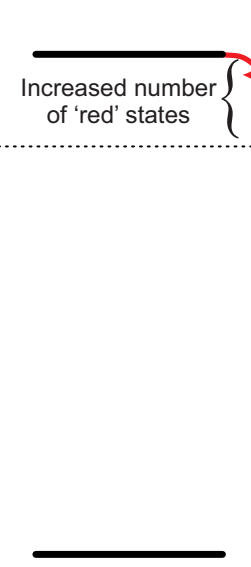

Chl

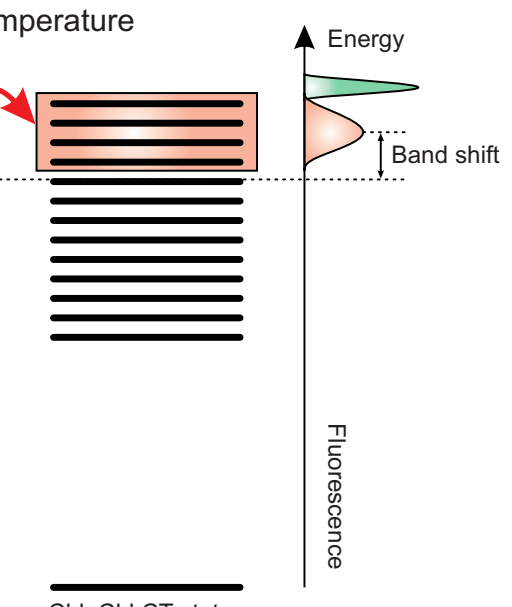

$\widehat{\text { Chl-Chl CT states }}$

Extended Data Figure 8 I Chlorophyll-chlorophyll charge transfer state can explain both the blue shift of the fluorescence peak as well as the increased concentration of the red-emitting states at lower temperature. Depending on local environmental conditions, numerous Chl-Chl CT states with varying energy can be formed in distinct LHCII complexes, but only some of them are mixed with other Chl excitonic states and can collect excitation coming from the antenna. Strong interactions with the unharmonic environment at lower temperatures can induce blue shift of all the CT states, ${ }^{29}$ so that CT states become mixed with the Chl exciton states in a larger number of LHCII complexes. This results in the blue-shifted peak position of the fluorescence spectrum ( $c f$. Figure 2b) of red component as well as in the increased concentration of the red states per aggregate ( $c f$. Figure $6 \mathrm{~d})$. 

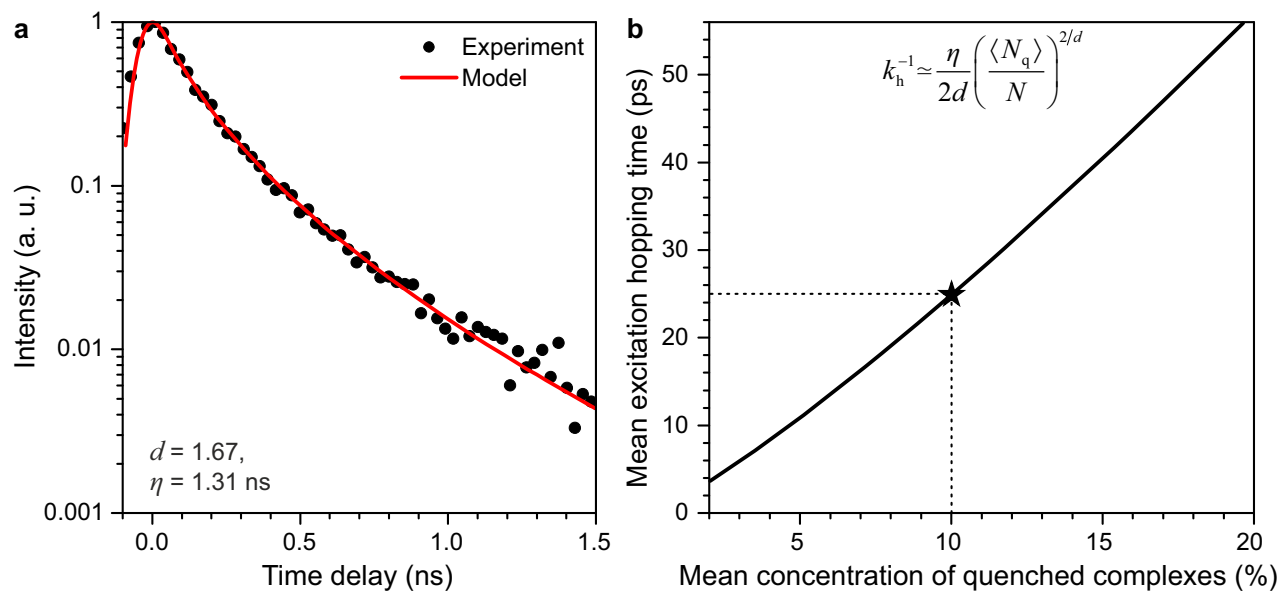

Extended Data Figure 9 । Fluorescence decay kinetics in the LHCII aggregate, calculated using the fluctuating antenna model. ${ }^{21,48}$ a, fitted fluorescence kinetics at $273 \mathrm{~K}$ and the obtained model parameters. b, the obtained relationship between the mean inter-complex excitation hopping time, $k_{\mathrm{h}}^{-1}$, and the mean relative number of the quenched complexes per aggregate, $\left\langle N_{\mathrm{q}}\right\rangle / N$. Star indicates the mean hopping time of $25 \mathrm{ps}$, corresponding to 1 trap per 10 LHCII monomers. 